\title{
Adaptación psicométrica de las Escalas de Afrontamiento para Adolescentes de Frydenberg y Lewis en un grupo de escolares de Lima metropolitana
}

Beatriz Canessa

Universidad de Lima

Lima, Perú

El presente estudio tuvo como objetivo la adaptación psicométrica de la prueba Escalas de Afrontamiento para Adolescentes (ACS) de Frydenberg y Lewis. Se trabajó con 1.236 escolares de ambos sexos, con edades entre los 14 y los 17 años, de los niveles socioeconómicos alto, medio y bajo. Los resultados demostraron que todos los puntajes de las ACS Lima eran confiables. Así mismo, se llegó a la conclusión de que dicha prueba posee validez de constructo, ya que las correlaciones item-test en cada una de las escalas obtuvieron valores significativos, y los análisis factoriales realizados revelaron que las 18 escalas que conforman el ACS se mantienen en la versión adaptada para nuestro medio, con la inclusión, en algunos casos, de items especificos.

\section{Psychometric adaptation of the Frydenberg and Lewis test "Adolescent Coping Scales" to a group of school students in metropolitan Lima}

The aim of the current study was to perform a psychometric adaptation of the Frydenberg and Lewis's test "Adolescent Coping Scales" (ACS). Participants were 1.236 students, male and female, from different socio-economic level, high, middle and low. Their ages ranged from 14 to 17 years old. Results of all the scores for the ACS Lima were shown to be reliable. It was also concluded that this test has construct validity, due to the item-test correlation for every scales demonstrataed to be significant, and factor analysis showed that the 18 scales that form the ACS are to conform the adapted version of this test for our environment, including specific items in some cases.

psychometric adaptation / coping / adolescence

Dirección de la autora: bcanessa@correo.ulima.edu.pe 
El afrontamiento ha ido cobrando cada vez mayor relevancia en el campo de la psicología, pues el interés por conocer cómo las personas afrontan sus problemas y los factores que determinan el manejo de las diferentes preocupaciones ha ido en aumento, comenzando así a ser investigado alrededor de los años sesenta, con poblaciones adultas, y extendiéndose recientemente su estudio a niños y adolescentes (Lazarus y Folkman, 1986; Ayers, Sandler y Thowey, 1998).

La idea principal que, según Frydenberg (1997a), motiva la investigación sobre el afrontamiento está en la creencia de que nuestras acciones frente al estrés y los recursos que tenemos para manejarlo determinan en gran parte el proceso de aprendizaje y de desarrollo del individuo, así como también su calidad de vida. Es por esta razón que el afrontamiento es considerado como una competencia psicosocial, que incluye una serie de estrategias conductuales y cognitivas utilizadas para enfrentar las demandas de la vida (Frydenberg y Lewis, 1994; 1999).

El interés por realizar la presente investigación reside en el hecho de que las diferentes problemáticas que presenta la adolescencia de nuestro país, como son el pandillaje, el consumo de drogas o los suicidios, podrían ser mejor comprendidas e intervenidas, si se conociera qué estrategias de afrontamiento utilizan los adolescentes frente a sus dificultades.
A lo anterior se añade la necesidad de adaptar pruebas psicométricas a nuestro medio para, de esta manera, tener a disposición herramientas que permitan evaluar cada vez más distintos aspectos de la conducta en diversas poblaciones, como es en este caso la población adolescente. Esto permitirá contar con variados instrumentos que sean acordes con nuestra realidad, y además útiles no sólo para el campo de la investigación, sino también para el campo de la evaluación y el tratamiento.

La presente investigación ofrece un nuevo instrumento dirigido a evaluar las conductas de afrontamiento en el adolescente, lo cual es de especial importancia debido al número limitado de instrumentos válidos y confiables diseñados para la evaluación de tales conductas (Boekaerts, 1996).

\section{ESTRÉS Y AFRONTAMIENTO}

El marco teórico de los estudios llevados a cabo por Frydenberg está basado en el modelo sobre el estrés y el afrontamiento desarrollado por Lazarus, así como en las investigaciones llevadas a cabo con el instrumento Adolescent Coping Scale, elaborado por Frydenberg y Lewis en 1993.

El modelo transaccional de Lazarus enfatiza la interacción entre la persona y el ambiente, por lo tanto, el estrés es concebido como un desbalance entre la percepción de la persona de las demandas que se le imponen y la percepción de los recursos que ella tiene para 
afrontar estas demandas, evaluándose el entorno como amenazante o desbordante de sus recursos. Es la experiencia subjetiva la que determina si un evento es estresante o no (Lazarus y Folkman, 1986; Frydenberg y Lewis, 1993a; Sandín, 1995).

Además, Lazarus y Folkman (1986) consideran el afrontamiento como aquellos esfuerzos cognitivos y conductuales constantemente cambiantes que se desarrollan para manejar las demandas específicas externas y/o internas que son evaluadas como excedentes o desbordantes de los recursos del individuo. Tales autores consideran el afrontamiento como un proceso, lo que implica asumir los siguientes principios (Lazarus, 1993a, 1993b; Sandín, 1995; Frydenberg, 1997a):

- El afrontamiento depende del contexto y, por lo tanto, puede cambiar durante una situación determinada.

- Los pensamientos y las acciones del afrontamiento se definen por el esfuerzo y no por el éxito.

- La teoría del afrontamiento como un proceso enfatiza que existen al menos dos funciones principales, una orientada al problema y otra orientada a la emoción, cuya utilización depende del grado de control que se tenga sobre la situación.

- Algunas estrategias de afrontamiento son más estables o consistentes en situaciones estresantes que otras.

- El afrontamiento es capaz de mediar el resultado emocional, modifican- do el estado emocional del inicio al fin en un encuentro estresante.

El desarrollo teórico y metodológico del afrontamiento ha llevado a que éste sea concebido desde diferentes enfoques, los cuales pueden ser agrupados, principalmente, en dos aproximaciones distintas. Una de ellas, denominada afrontamiento disposicional, asume que el afrontamiento es un estilo personal de afrontar el estrés, mientras que la otra, conocida como afrontamiento situacional, entiende el afrontamiento como un proceso, como una reacción al estrés de carácter dinámico y específico para la situación (Sandín, 1995). Frydenberg y Lewis (1993a, 1994, 1997a) consideran esta distinción en su conceptualización y evaluación del afrontamiento refiriéndose, respectivamente, a los elementos generales y específicos del constructo. Es por esto que su instrumento Adolescent Coping Scale (ACS) cuenta con una forma general que permite determinar cómo los individuos suelen afrontar sus preocupaciones, y con una forma específica que permite evaluar las respuestas de afrontamiento frente a un problema determinado.

Otra forma de categorizar el afrontamiento va desde el enfoque dicotómico de Lazarus y Folkman, quienes identifican el afrontamiento orientado al problema y el afrontamiento orientado a la emoción, hasta la especificación de 18 estrategias de afrontamiento, como se hace en el instrumento desarrollado por 
Frydenberg y Lewis. Dichas estrategias han sido, además, agrupadas en tres estilos de afrontamiento, los cuales pueden representar aspectos funcionales o disfuncionales de afrontamiento. El afrontamiento funcional o adaptativo es conceptualizado como aquél en el que el problema es definido, las alternativas de solución generadas y las acciones son llevadas a cabo; mientras que el afrontamiento disfuncional se refiere al manejo y la expresión de sentimientos al momento de afrontar eventos estresantes. Ambos tipos dependen de la bondad del ajuste entre la evaluación que la persona hace de lo que está sucediendo y de sus opciones de afrontamiento (Frydenberg, 1997a).

Dos son los estilos considerados como productivos o funcionales, uno de ellos es el denominado Resolver el problema, que refleja la tendencia a abordar las dificultades de manera directa, y el otro es el de Referencia hacia los otros, que implica compartir las preocupaciones con los demás y buscar soporte en ellos.

El Afrontamiento no-productivo es el estilo disfuncional, ya que las estrategias pertenecientes a éste no permiten encontrar una solución a los problemas, orientándose más bien a la evitación (Frydenberg y Lewis, 1993a; 1996a; 1999).

\section{AFrontamiento adolescente: LA PROPUESTA DE EVALUACIÓN DE FrYdenberG}

Aunque la mayoría de los esfuerzos en la conceptualización, evaluación e investigación del afrontamiento se han llevado a cabo con poblaciones adultas, ha habido un incremento en el número de estudios sobre este tema con niños y adolescentes (Ayers et al., 1998).

Las primeras nociones de afrontamiento en la adolescencia surgieron alrededor de los años setenta sobre la base de la literatura teórica y empírica existente sobre estrés y afrontamiento en la adultez. El trabajo con adultos proveyó los modelos conceptuales y las herramientas metodológicas para la investigación con adolescentes; sin embargo, luego de algunos años, los investigadores se preguntaron qué tanto los modelos y resultados encontrados con los adultos podían ser generalizables a poblaciones más jóvenes, lo cual llevó a centrar los estudios en el afrontamiento propio de la adolescencia (Hoffman et al., 1992).

Las diversas investigaciones en este campo se han centrado no sólo en describir las estrategias de afrontamiento, sino también en conocer los eventos estresores más comunes en esta etapa de la vida, intentándose así tener una visión más clara y profunda sobre el tema del estrés y el afrontamiento en la adolescencia. 
Varios han sido los métodos empleados para elaborar diversos instrumentos, los que tienen como objetivo evaluar el afrontamiento en la adolescencia, tanto desde la perspectiva situacional como desde la disposicional (Boekaerts, 1996). Uno de estos instrumentos es el desarrollado por Frydenberg y Lewis en 1993, instrumento que a continuación se describe junto con los principales planteamientos propuestos por Frydenberg sobre el afrontamiento adolescente.

A partir de su trabajo con adolescentes, Frydenberg y Lewis definen el afrontamiento adolescente como:

... un conjunto de acciones cognitivas y afectivas que surgen en respuesta a una preocupación en particular. Ellas representan un intento por restaurar el equilibrio o reducir la turbulencia para el individuo. Esto puede hacerse resolviendo el problema, es decir, cambiando el estímulo, o acomodándose a la preocupación sin necesariamente dar una solución (Frydenberg, 1993a, 255).

El afrontamiento es considerado, entonces, como las conductas y acciones que se generan en respuesta a las demandas planteadas al individuo. Algunas de estas acciones intentan modificar o remediar la fuente de la demanda (por ejemplo, resolver el problema), otras ayudan al individuo a acomodarse a ésta (por ejemplo, hacerse ilusiones), y otras indican cierta incapacidad para manejar la demanda (por ejemplo, desesperarse y enfermarse) (Frydenberg y Lewis, 1999).
El instrumento Adolescent Coping Scale (ACS), elaborado por Frydenberg y Lewis en 1993 se constituyó como una alternativa para el estudio del afrontamiento en el adolescente. Éste consta de 79 ítems cerrados más uno abierto, los cuales identifican las estrategias de afrontamiento más comunes utilizadas por los adolescentes. Los ítems se distribuyen en 18 escalas, cada una de las cuales refleja una respuesta de afrontamiento diferente y contiene entre 3 y 5 ítems.

Las 18 escalas del ACS han sido rotuladas de tal manera que reflejen el constructo inherente en los ítems. Éstas son las siguientes (Frydenberg y Lewis, 1997b; 1999):

- Buscar apoyo social (As).- Estrategia que consiste en la inclinación a compartir el problema con otros y buscar apoyo para su resolución. Ejemplo: "Hablar con otros sobre mi problema para que me ayuden a salir de él".

- Concentrarse en resolver el problema $(R p)$.- Es una estrategia dirigida a resolver el problema, estudiándolo sistemáticamente y analizando los diferentes puntos de vista u opciones. Ejemplo: "Dedicarme a resolver el problema poniendo en juego todas mis capacidades".

- Esforzarse y tener éxito (Es).- Es la estrategia que comprende conductas que ponen de manifiesto compromiso, ambición y dedicación. Ejemplo: "Trabajar intensamente". 
- Preocuparse (Pr).- Se caracteriza por elementos que indican temor por el futuro en términos generales $o$, más en concreto, preocupación por la felicidad futura. Ejemplo: "Preocuparme por lo que está pasando".

- Invertir en amigos íntimos (Ai).- Se refiere al esfuerzo por comprometerse en alguna relación de tipo personal íntimo e implica la búsqueda de relaciones personales íntimas. Ejemplo: "Pasar más tiempo con el chico o chica con que suelo salir".

- Buscar pertenencia (Pe).- Indica la preocupación e interés del sujeto por sus relaciones con los demás en general $\mathrm{y}$, más concretamente, por lo que otros piensan. Ejemplo: "Mejorar mi relación personal con los demás".

- Hacerse ilusiones (Hi).- Es la estrategia expresada por ítems basados en la esperanza, en la anticipación de una salida positiva y en la expectativa de que todo tendrá un final feliz. Ejemplo: "Esperar a que ocurra algo mejor".

- Falta de afrontamiento (Na).- Consiste en ítems que reflejan la incapacidad del sujeto para enfrentarse al problema y su tendencia a desarrollar síntomas psicosomáticos. Ejemplo: "No tengo forma de afrontar la situación".

- Reducción de la tensión (Rt).- Se caracteriza por ítems que reflejan un intento por sentirse mejor y de relajar la tensión. Ejemplo: "Intentar sentirme mejor bebiendo alcohol, fumando o tomando drogas".

- Acción social (So).- Consiste en dejar que otros conozcan cuál es el problema y tratar de conseguir ayuda escribiendo peticiones u organizando actividades como reuniones o grupos. Ejemplo: "Unirme a gente que tiene el mismo problema".

- Ignorar el problema (Ip).- Agrupa ítems que reflejan un esfuerzo consciente por negar el problema o desentenderse de él. Ejemplo: "Ignorar el problema".

- Autoinculparse (Cu).- Incluye conductas que indican que el sujeto se ve como responsable de los problemas o preocupaciones que tiene. Ejemplo: "Sentirme culpable".

- Reservarlo para sí (Re).- Es la estrategia expresada por ítems que reflejan que el sujeto huye de los demás y no desea que conozcan sus problemas. Ejemplo: "Guardar mis sentimientos para mí solo".

- Buscar apoyo espiritual (Ae).- Está compuesta por ítems que reflejan una tendencia a rezar, a emplear la oración y a creer en la ayuda de un líder espiritual o Dios. Ejemplo: "Dejar que Dios se ocupe de mi problema".

- Fijarse en lo positivo (Po).- Se caracteriza por ítems que indican una visión optimista y positiva de la situación presente y una tendencia a ver el lado bueno de las cosas y considerarse afortunado. Ejemplo: "Fi- 
jarme en el aspecto positivo de las cosas y pensar en las cosas buenas".

- Buscar ayuda profesional (Ap).- Es la estrategia consistente en buscar la opinión de profesionales, como maestros u otros consejeros. Ejemplo: "Pedir consejo a una persona competente".

- Buscar diversiones relajantes (Dr).Se caracteriza por ítems que describen actividades de ocio y relajantes, como leer o pintar. Ejemplo: "Encontrar una forma de relajarme; por ejemplo, oír música, leer un libro, tocar un instrumento musical, ver la televisión".

- Distracción física (Fi).- Consta de ítems que se refieren a la dedicación al deporte, al esfuerzo físico y a mantenerse en forma. Ejemplo: "Mantenerme en forma y con buena salud".

Estas 18 escalas o estrategias de afrontamiento son agrupadas en tres estilos de afrontamiento, cada uno de los cuales comprende entre cuatro y ocho estrategias. El primero de estos estilos, llamado Resolver el problema (Solve the problem), está caracterizado por los esfuerzos que se dirigen a resolver el problema manteniendo una actitud optimista y socialmente conectada. Este primer estilo comprende las estrategias Concentrarse en resolver el problema, Esforzarse y tener éxito, Invertir en amigos íntimos, Buscar pertenencia, Reservarlo para sí, Fijarse en lo positivo, Buscar diversiones relajantes y
Distracción física. El segundo estilo se denomina Referencia a otros (Reference to others) e implica un intento para enfrentar el problema acudiendo al apoyo y a los recursos de las demás personas, como pares, profesionales o deidades. Las estrategias Buscar apoyo social, Acción social, Buscar apoyo espiritual y Buscar ayuda profesional pertenecen a este estilo. El último estilo, el Afrontamiento no productivo (Non-productive coping), está asociado a una incapacidad para afrontar los problemas e incluye estrategias de evitación. Tal estilo no lleva a la solución del problema, pero al menos lo alivia. Comprende las siguientes estrategias: Preocuparse, Buscar pertenencia, Hacerse ilusiones, Falta de afrontamiento o No afrontamiento, Ignorar el problema, Reducción de la tensión, Reservarlo para sí, y Autoinculparse (Frydenberg y Lewis, 1993a; Frydenberg, 1997a).

Una vez obtenido el instrumento, Frydenberg y colaboradores realizaron una serie de investigaciones, que, además de estar dirigidas a analizar sus niveles de validez y confiabilidad, han permitido conocer, describir y comparar las estrategias de afrontamiento utilizadas por los adolescentes. Así, se han interesado por estudiar la relación del género, la edad y el estrato socioeconómico con el afrontamiento adolescente (Frydenberg y Lewis, 1991; 1993a; 1997a; 1999). Además, han investigado las diferentes preocupacio- 
nes que acompañan a los adolescentes y la forma como las enfrentan, especialmente las vinculadas con temas sociales (Frydenberg y Lewis, 1996b; Frydenberg y Rowley, 1998); el afrontamiento en adolescentes talentosos (Frydenberg, 1993b; 1997a); la relación entre afrontamiento, temperamento, autoconcepto y depresión en adolescentes, así como la influencia de la familia y el soporte social en las estrategias de afrontamiento (Frydenberg, 1997a). En la siguiente sección se desarrollan tales investigaciones vinculándolas con otras realizadas por investigadores pertenecientes a este campo del afrontamiento.

\section{Validez y confiabilidad de la prueba Adolescent Coping Scale (versión original)}

Las descripciones sobre las conductas de afrontamiento dadas por un grupo de 2.041 adolescentes australianos dieron las bases para el desarrollo de este instrumento. A través de dos estudios, uno en 1990 (643 estudiantes australianos, de ambos sexos, entre los 15 y los 18 años de edad) y otro en 1992 (576 escolares, de ambos sexos, entre los 13 y los 17 años de edad), Frydenberg y Lewis (1996a, 1997b) verificaron la validez y confiabilidad del mencionado instrumento.

En ambos estudios se utilizaron las respuestas de afrontamiento dadas por los estudiantes frente a una preocupación particular autonombrada, ya que si éstas están asociadas con un problema específico tienden a demostrar más que las respuestas generales dadas por los estudiantes, la existencia de un número separado de factores o escalas conceptualmente interpretables. En el primer estudio, se les pidió a los estudiantes nombrar sus mayores preocupaciones, mientras que en el segundo se les pidió que se restringieran a temas relacionados con la familia (Frydenberg y Lewis, 1996a).

Para analizar la validez de constructo, es decir hasta qué punto los ítems evalúan las 18 estrategias de afrontamiento, diferentes pero relacionadas entre sí, se utilizó el análisis factorial a través del método de los componentes principales con rotación oblicua oblimin. La rotación oblicua fue usada debido a la idea de que las subescalas podrían no ser enteramente ortogonales o independientes. Es decir, se planteó la hipótesis de que, aunque el afrontamiento puede considerarse como un conjunto de diversas áreas o estrategias, cada una con diferentes tipos de acciones, esas estrategias no deberían considerarse como carentes de relación entre sí.

Para realizar este análisis se agruparon, en primer lugar, los ítems o elementos en función de las escalas para las que se habían elaborado. Luego, los 18 grupos se asignaron aleatoriamente a tres categorías cada una de las cuales incluía todos los elementos correspondientes a seis estrategias diferentes. Por 
último, se hicieron tres análisis factoriales en cada grupo de ítems de las categorías respectivas, usando las respuestas dadas por los sujetos de la muestra (Frydenberg y Lewis, 1996a; 1997b).

Luego de realizar estos tres análisis factoriales, se encontró que las 18 escalas tuvieron validez de constructo, apoyándose así la existencia de dichas dimensiones. Con respecto a la confiabilidad, se encontró que en ambos estudios las respuestas a los ítems tuvieron suficiente estabilidad. Solamente en dos escalas de la forma específica, Buscar diversiones relajantes y Distracción física, la fiabilidad interna fue inferior a $.65 \mathrm{y}$ en ambos casos se trata de escalas con sólo tres reactivos. Esto pudo deberse, según los autores, a que los sujetos no consideraron la forma de afrontar un problema concreto, sino la forma de afrontar dificultades en un contexto más general (Frydenberg y Lewis, 1996a; 1997b).

El ACS alcanzó coeficientes de consistencia interna que oscilaron entre .62 y $.87(\mathrm{M}=.73)$ en la forma específica, y entre.54 y .84 $(\mathrm{M}=.71)$ en la forma general (Frydenberg, 1997a).

Además, se encontró que las correlaciones test-retest fueron más moderadas que altas. Alrededor del 50\% de los coeficientes fueron inferiores a .70 tanto en la forma general como en la forma específica. Sin embargo, hay que tener en cuenta que la estabilidad de respuesta no es enteramente una mane- ra apropiada de evaluar la confiabilidad de las respuestas de afrontamiento de los estudiantes, desde que el afrontamiento es considerado como un fenómeno dinámico. Las confiabilidades test-retest para las subescalas variaron de .49 a $.82(\mathrm{M}=.68)$ en la forma específica, y de .44 a $.84(\mathrm{M}=.69)$ en la forma general (Frydenberg y Lewis, 1996; 1997a).

A partir de lo revisado, se llevó a cabo una investigación de tipo psicométrica, con el fin de realizar la estandarización de las Escalas de Afrontamiento para Adolescentes (ACS) en un grupo de escolares de diferente edad, sexo y nivel socioeconómico.

\section{OBJetivos}

\section{Objetivo general}

Realizar la adaptación psicométrica de las Escalas de Afrontamiento para Adolescentes (ACS) en una muestra de escolares de Lima metropolitana.

\section{Objetivos específicos}

- Determinar la validez de contenido de las Escalas de Afrontamiento para Adolescentes (ACS) a través del método del criterio de jueces.

- Determinar la validez de constructo de las Escalas de Afrontamiento para Adolescentes (ACS) basándose en el método intraprueba a través del análisis de ítems y el análisis factorial. 
- Determinar la confiabilidad de las Escalas de Afrontamiento para Adolescentes (ACS) a través del método de la consistencia interna por medio del coeficiente alfa de Cronbach.

\section{MÉTodo}

\section{Muestra}

La población utilizada para la presente investigación está representada por los alumnos de tercero, cuarto y quinto de secundaria pertenecientes a centros educativos estatales y particulares de Lima metropolitana. Esta población, según datos proporcionados por el INEI (1999), está conformada en total por un número de 638.791 alumnos. De éstos, 483.463 alumnos se distribuyen en 811 colegios estatales, y 155.328 se encuentran distribuidos en 1.204 colegios particulares.

La muestra estuvo conformada por 1.236 escolares. De ellos, 636 (51,5\%) fueron hombres y $600(48,5 \%)$ mujeres, con edades entre los 14 y los 17 años. Del total de sujetos, el 19,5\% tenía 14 años de edad; el 33,4\% reportó tener 15 años; el 31,2\% tenía 16 años; y el $15,9 \%, 17$ años de edad. Con respecto al grado de estudios, el $38,3 \%$ se encontraba cursando tercero de secundaria; el $31 \%$, cuarto de secundaria, y el $30,7 \%$, quinto de secundaria.

En relación con el nivel socioeconómico, el $26,9 \%$ de los escolares pertenecía al estrato alto; el $42,8 \%$ se ubicó en el estrato socioeconómico medio; y el 30,3\% pertenecía al nivel socioeconómico bajo.

Para reunir a los sujetos, se seleccionaron los colegios, a partir de la clasificación económica hecha por la Universidad de Lima, cuyo parámetro principal es el costo promedio de la pensión mensual. Los colegios seleccionados fueron doce: cuatro de ellos pertenecientes al nivel socioeconómico alto, los otros cuatro al nivel socioeconómico medio y los restantes al nivel socioeconómico bajo.

Se utilizó una ficha socioeconómica individual, a partir de la cual se elaboró un indicador socioeconómico, que permitió establecer que la categorización económica de los colegios hecha por la Universidad de Lima era representativa de los niveles socioeconómicos. Este indicador estuvo formado por seis aspectos: grado de instrucción del encargado(a) de la familia; grado de instrucción de la pareja del encargado(a) de la familia; el material predominante en las paredes de la vivienda; el material predominante en los pisos de la vivienda; el nivel de hacinamiento, determinado por la proporción entre el número de habitaciones y el número de personas que viven en la casa; y el número de artefactos electrodomésticos.

Es importante mencionar que dicha muestra fue seleccionada siguiendo las pautas del diseño muestral no probabilístico de tipo intencional, lo cual se constituye como la principal limitación 
de dicha investigación. Sin embargo, los hallazgos obtenidos pueden ser generalizados a aquellos adolescentes que presenten características similares a la muestra utilizada.

\section{Instrumentos}

\section{Ficha socioeconómica}

Esta ficha fue elaborada con el fin de obtener datos personales y sociodemográficos sobre los sujetos participantes y ubicar el nivel socioeconómico al que éstos pertenecían.

Para la elaboración de esta ficha se revisaron los estudios sobre Niveles socioeconómicos efectuados por Apoyo Opinión y Mercado S.A. (1999) y la encuesta elaborada por Cedro para su investigación sobre Epidemiología de drogas en la población urbana peruana, 1997 (1999).

\section{Escalas de Afrontamiento para}

Adolescentes (Adolescent

Coping Scale, ACS)

El ACS fue elaborado por Frydenberg y Lewis (1993) en Australia, y luego adaptado por Pereña y Seisdedos (1995) en España, siendo esta adaptación la utilizada en la presente investigación. El ACS es un instrumento tanto de investigación como de terapia y orientación, que permite a los jóvenes examinar sus propias conductas de afrontamiento.
El instrumento se aplica a adolescentes entre 12 y 18 años de edad, aunque la experiencia ha demostrado que puede ser utilizado con personas mayores que este rango de edad.

El ACS puede ser administrado en forma individual y colectiva. Normalmente, se tarda unos 10 minutos en contestar el inventario y el tiempo total de aplicación, incluyendo las instrucciones, y la explicación del objetivo de la prueba no suele superar los 15 minutos.

El ACS es un inventario de autoinforme compuesto por 80 reactivos, 79 de tipo cerrado y uno final abierto, que permite evaluar con fiabilidad 18 escalas o estrategias de afrontamiento que se han identificado conceptual y empíricamente. Los 79 reactivos cerrados se puntúan mediante una escala de tipo Likert de cinco puntos:

- No me ocurre nunca o no lo hago.

- Me ocurre o lo hago raras veces.

- Me ocurre o lo hago algunas veces.

- Me ocurre o lo hago a menudo.

- Me ocurre o lo hago con mucha frecuencia.

El ACS consta de dos formas: una forma especifica, que permite evaluar las respuestas a un problema particular citado por el sujeto o propuesto por el examinador; y una forma general, forma utilizada en esta investigación y que se refiere a cómo el sujeto afronta sus problemas en general. Ambas formas contienen los mismos ítems, aunque varían en las instrucciones de aplicación. 
En la versión original de la prueba existe también una forma abreviada de la escala con sólo 18 ítems, uno por cada subescala. Sin embargo, en la versión española no se ha considerado esta forma, ya que la escala completa es de simple aplicación y el tiempo requerido es muy breve.

Cada una de las 18 escalas del ACS tiene diferente número de reactivos. Así, las nueve primeras escalas cuentan con cinco reactivos, las siete siguientes tienen cuatro reactivos y las dos últimas sólo cuentan con tres reactivos.

Estas 18 escalas se agrupan en tres estilos básicos de afrontamiento, ya descritos anteriormente:

- Estilo 1: Dirigido a Resolver el problema (Solve the problem).

- Estilo 2: Afrontamiento no productivo (Non-productive coping).

- Estilo 3: Referencia a otros (Reference to others).

Los pasos a seguir para la corrección del ACS son los siguientes:

- Obtener la puntuación total para cada escala, sumando los puntos obtenidos en cada uno de los cinco, cuatro o tres ítems de que consta la escala, y anotarla en la casilla de puntuación total.

- Si algún sujeto ha dejado algún ítem en blanco se le pedirá que complete la prueba y si ello no es posible se puntuará ese ítem como tres porque ello implica menor distorsión de los resultados que dejarlo en blanco y, por lo tanto, valorarlo con un cero.
Si el sujeto ha dejado dos o más elementos en blanco en una escala, se debe dejar dicha escala sin valorar.

- Las respectivas puntuaciones totales se multiplicarán por el coeficiente indicado en la hoja de respuestas y el resultado se anotará en la casilla Puntuación ajustada.

- Las puntuaciones ajustadas pueden trasladarse a la hoja de perfil.

Adaptación española de la prueba Adolescent Coping Scale (ACS)

Se trabajó con una muestra de 452 sujetos, compuesta por 162 (36\%) varones y $290(64 \%)$ mujeres. Del total de sujetos, $47(10 \%)$ tenían entre 12 y 14 años de edad; 217 (48\%) tenían entre 15 y 17 años de edad; y 188 (41\%) tenían entre 18 y 20 años de edad.

Con relación al análisis de elementos, se observó, en primer lugar, que tanto hombres como mujeres se inclinan de manera parecida por negar (respuesta Nunca) o afirmar (respuesta Con mucha frecuencia) los contenidos de las cuestiones. Además, se encontró que el primero de los elementos con media más alta fue el de Asistir a clase con regularidad, en donde el promedio obtenido por ambos sexos supera la alternativa A menudo y el de las mujeres se acerca aún más al valor tope de la escala. En el otro polo, el elemento con una media más baja fue Leer un libro sagrado o de religión. 
Los elementos con mayor atracción a favor de los varones fueron: Hacer deporte, Mantenerse en forma e Ir al gimnasio, y los elementos con mayor atracción a favor de las mujeres fueron: Llorar o gritar y Tener dolores de cabeza.

Por otra parte, se analizó también la capacidad discriminativa de los elementos y se identificaron los dos elementos con mayor heterogeneidad y los dos elementos con mayor homogeneidad. A partir de los resultados de la muestra, dos conductas fueron las que presentaron gran dispersión entre los adolescentes: Guardar los sentimientos y Pasar más tiempo con el chico o la chica con el/la que suelo salir. En el otro extremo, dos fueron las conductas: Leer un libro sagrado o de religión y Organizar un grupo que se ocupe del problema, en las que hubo una gran unanimidad en las respuestas.

Finalmente, se analizaron las correlaciones entre las subescalas del ACS y para esto se calcularon las intercorrelaciones en la muestra total $(\mathrm{N}=452)$ y la matriz resultante fue sometida a un análisis factorial de componentes principales. Como resultado de este análisis, se definieron cuatro grandes dimensiones claramente diferenciadas:

- Se busca una acción positiva y esforzada, y en este factor intervienen las subescalas Concentrarse en resolver el problema (Rp), Esforzarse y tener éxito (Es), Preocuparse (Pr) y Atacar el problema (peso negativo en Ip, Ignorar el problema).
- Hay una huida intropunitiva, definida por las subescalas de No afrontamiento $(\mathrm{Na})$, Reducción de la tensión (Rt) y Autoinculparse $(\mathrm{Cu})$.

- Una acción positiva hedonista, cuyos componentes son: Buscar diversiones relajantes (Dr), Fijarse en lo positivo (Po), Hacerse ilusiones (Hi), Buscar pertenencia (Pe), Invertir en amigos íntimos (Ai), Ignorar el problema (Ip) y Distracción física (Fi).

- Una postura de introversión, en la que el problema se Reserva para sí (Re), y se evitan (pesos negativos en las tres subescalas siguientes) la Acción social (So), Buscar ayuda profesional (Ap) y Buscar apoyo social (As).

Los índices obtenidos en el análisis parecen mostrar que hay una relación negativa (-.28) entre una acción positiva y esforzada (factor I) y la postura de introversión (factor IV), así como entre esta última y la acción positiva hedonista (-.19). Sin embargo, la relación entre las dos acciones positivas, la esforzada y la hedonista, es positiva (.19). (Frydenberg y Lewis, 1997b).

\section{Procedimiento}

Como ya se mencionó, la adaptación psicométrica se llevó a cabo con la versión española del Adolescent Coping Scale (ACS) en su forma general. Previamente dicha versión fue comparada con la versión original en inglés para verificar si mantenía la misma estruc- 
tura y las mismas características, encontrándose que no se han producido modificaciones de redacción lingüística considerables.

En primer lugar, se llevó a cabo la adaptación lingüística de la prueba, para lo cual fue revisada por 10 jueces, todos psicólogos profesionales. Una vez que se tuvieron las evaluaciones hechas por los jueces, se aplicó la fórmula del coeficiente $V$ de Aiken (Escurra, 1988) para identificar qué ítems debían ser modificados según sus sugerencias.

Después de hacer los cambios respectivos de acuerdo con los requerimientos observados, se elaboró la versión revisada, la cual fue aplicada a un grupo piloto de 19 escolares de centros educativos particulares y estatales, entre los 13 y los 18 años (9 pertenecientes a un nivel socioeconómico alto y 9 pertenecientes a un nivel socioeconómico bajo; de todos ellos 6 fueron hombres y 13 mujeres). Ésta se llevó a cabo para ver si se presentaba algún tipo de dificultad en los ítems revisados.

Luego de dicha aplicación, se realizaron modificaciones en las instrucciones, la redacción de los ítems y las alternativas de respuesta, obteniéndose así la versión final, a la que se le dio el nombre de Escalas de Afrontamiento para Adolescentes Revisadas (ACS Lima), (véase anexo 1).

Una vez elaborada dicha versión, se procedió a contactar los 12 colegios previamente seleccionados, pertene- cientes a tres niveles socioeconómicos diferentes: alto, medio y bajo.

La administración se realizó de manera colectiva y, en general, tomó un tiempo aproximado de 30 minutos.

La secuencia de la aplicación fue la siguiente: en primer lugar, se administró la ficha socioeconómica, y en segundo lugar, la prueba Escalas de Afrontamiento para Adolescentes Revisadas (ACS Lima).

\section{Resultados}

Para el análisis psicométrico de los resultados de la investigación, se presenta el cálculo de la validez de constructo realizado a partir del análisis de ítems de la prueba Escalas de Afrontamiento para Adolescentes Revisadas (ACS Lima), el análisis factorial de los ítems, y el análisis factorial de las escalas del ACS Lima. Además, se presenta el análisis de la confiabilidad por consistencia interna.

\section{Análisis de ítems del ACS Lima}

Para aceptar los ítems se tuvieron en cuenta dos criterios: en primer lugar, que la correlación ítem-test corregido fuera mayor de 0.20; y en segundo lugar, que la presencia del ítem, a pesar de que pudiera tener una correlación ítem-test corregido inferior a 0.20 , incrementara el coeficiente alfa de Cronbach. 
Tabla $N^{\circ} 1$

Análisis de ítems de la escala 1 a la escala 6

\begin{tabular}{|c|c|c|c|}
\hline Escalas & Ítem & M & ritc \\
\hline \multirow[t]{5}{*}{ 1. Buscar apoyo social (As) } & 01 & 2.9 & $.50 *$ \\
\hline & 20 & 3.0 & .58 * \\
\hline & 38 & 3.3 & .40 * \\
\hline & 56 & 2.8 & .66 * \\
\hline & 72 & 3.1 & $.53^{*}$ \\
\hline \multirow[t]{5}{*}{ 2. Concentrarse en resolver el problema (Rp) } & 02 & 3.4 & .49 * \\
\hline & 21 & 3.5 & .56 * \\
\hline & 39 & 3.2 & .41 * \\
\hline & 57 & 3.5 & $.47^{*}$ \\
\hline & 73 & 3.6 & .56 * \\
\hline \multirow[t]{5}{*}{ 3. Esforzarse y tener éxito (Es) } & 03 & 3.4 & .44 * \\
\hline & 22 & 4.7 & $.13^{*}$ \\
\hline & 40 & 2.5 & .35 * \\
\hline & 58 & 4.0 & .32 * \\
\hline & 74 & 2.6 & .36 * \\
\hline \multirow[t]{5}{*}{ 4. Preocuparse (Pr) } & 04 & 4.0 & $.40 *$ \\
\hline & 23 & 4.1 & .36 * \\
\hline & 41 & 3.9 & .34 * \\
\hline & 59 & 3.9 & $.45^{*}$ \\
\hline & 75 & 3.1 & .32 * \\
\hline \multirow[t]{5}{*}{ 5. Invertir en amigos íntimos (Ai) } & 05 & 3.7 & .46 * \\
\hline & 24 & 3.2 & .49 * \\
\hline & 42 & 2.7 & $.48^{*}$ \\
\hline & 60 & 3.1 & $.40 *$ \\
\hline & 76 & 3.5 & $.55^{*}$ \\
\hline \multirow[t]{5}{*}{ 6. Buscar pertenencia $(\mathrm{Pe})$} & 06 & 3.9 & $.41 *$ \\
\hline & 25 & 3.6 & $.50 *$ \\
\hline & 43 & 3.3 & .45 * \\
\hline & 61 & 3.6 & $.57^{*}$ \\
\hline & 77 & 2.0 & .16 * \\
\hline
\end{tabular}

$\left(^{*}\right)$ Ítem aceptado

$\mathrm{N}=1236$

En la escala 1, Buscar apoyo social, se observa que los valores promedio de los ítems fluctuaron entre 2.8 para el ítem 56 y 3.3 para el ítem 38. En lo que se refiere a la correlación ítem-test corregido se puede ver que oscilaron entre .40 para el ítem 38 y .66 para el ítem
56, notándose que todos superaron los criterios mencionados anteriormente.

En la escala 2, Concentrarse en resolver el problema, los valores promedio de los ítems variaron entre 3.2 para el ítem 39 y 3.6 para el ítem 73; mientras que en la correlación ítem-test corregi- 
do lo hicieron entre .41 para el ítem 31 y .56 para los ítems 21 y 73 . Como puede verse, todos los ítems superaron los criterios establecidos.

Se puede observar también que los valores promedio de los ítems de la escala 3, Esforzarse y tener éxito, estuvieron entre 2.5 para el ítem 40 y 4.7 para el ítem 22. Respecto de la correlación ítem-test corregido, los valores estaban entre .13 para el ítem 22 y .44 para el ítem 03, superando todos los ítems los criterios indicados.

En la escala 4, Preocuparse, observamos que los valores promedio de los ítems fluctuaron entre 3.1 para el ítem 75 y 4.1 para el ítem 23. En lo que se refiere a la correlación ítem-test corregido, encontramos que éstos oscilaron entre .32 para el ítem 75 y .45 para el ítem 59, notándose que los ítems en su totalidad superaron los criterios considerados.

En la escala 5, Invertir en amigos íntimos, se puede ver que los valores promedio de los ítems variaron entre 2.7 para el ítem 42 y 3.7 para el ítem 05; mientras que en la correlación ítem-test corregido éstos se movieron entre .40 para el ítem 60 y .55 para el ítem 76 , encontrándose que todos los ítems cumplieron con los criterios establecidos.

Por último, en la escala 6, Buscar pertenencia, los valores promedio de los ítems oscilaron entre 2.0 para el ítem 77 y 3.9 para el ítem 06 . Con relación a la correlación ítem-test corregido, se puede observar que los valores varia- ron entre .16 para el ítem 77 y .57 para el ítem 61, superando todos los ítems los criterios mencionados.

En la tabla $\mathrm{N}^{\circ} 2$ se puede observar que en la escala 7, Hacerse ilusiones, los valores promedio de los ítems variaron entre 2.0 para los ítems 26 y 44 y 3.7 para los ítems 7 y 78 . En lo que se refiere a la correlación ítem-test corregido, se tiene que los valores se movieron entre .18 para el ítem 44 y .37 para el ítem 62, notándose que todos los ítems superan los criterios establecidos.

En la escala 8, Falta de afrontamiento o No afrontamiento, se encuentra que los valores promedio de los ítems fluctuaron entre 1.7 para el ítem 27 y 2.4 para el ítem 63; mientras que en la correlación ítem-test corregido, éstos oscilaron entre .25 para el ítem 8 y .40 para el ítem 63, observándose que todos los ítems cumplieron con los criterios señalados.

En la escala 9, Reducción de la tensión, se encuentra que los valores promedio de los ítems se movieron entre 1.5 para el ítem 28 y 2.5 para el ítem 64 , viéndose además que los valores para la correlación ítem-test corregido oscilaron entre .23 para el ítem 46 y .53 para el ítem 80, superando todos los ítems los criterios indicados.

Se encontró también que los valores promedio de los ítems de la escala 10 , Acción social, variaron entre 1.3 para el ítem 29 y 3.0 para el ítem 10 y que los valores de la correlación ítem-test corregido fluctuaron entre .18 para el 
Tabla No 2

Análisis de ítems de la escala 7 a la escala 12

\begin{tabular}{|c|c|c|c|}
\hline Escalas & Ítem & M & ritc \\
\hline \multirow[t]{5}{*}{ 7. Hacerse ilusiones $(\mathrm{Hi})$} & 07 & 3.7 & .24 * \\
\hline & 26 & 2.0 & .31 * \\
\hline & 44 & 2.0 & .18 * \\
\hline & 62 & 3.5 & .37 * \\
\hline & 78 & 3.7 & .36 * \\
\hline \multirow[t]{5}{*}{ 8. Falta de afrontamiento o no afrontamiento $(\mathrm{Na})$} & 08 & 2.2 & .25 * \\
\hline & 27 & 1.7 & .34 * \\
\hline & 45 & 1.8 & .35 * \\
\hline & 63 & 2.4 & $.40 *$ \\
\hline & 79 & 2.2 & .29 * \\
\hline \multirow[t]{5}{*}{ 9. Reducción de la tensión (Rt) } & 09 & 2.1 & .44 * \\
\hline & 28 & 1.5 & .35 * \\
\hline & 46 & 1.8 & .23 * \\
\hline & 64 & 2.5 & $.40 *$ \\
\hline & 80 & 2.0 & .53 * \\
\hline \multirow[t]{5}{*}{ 10. Acción social (So) } & 10 & 3.0 & $.18^{*}$ \\
\hline & 11 & 1.9 & .26 * \\
\hline & 29 & 1.3 & .33 * \\
\hline & 47 & 2.0 & $.40 *$ \\
\hline & 65 & 1.9 & .39 * \\
\hline \multirow[t]{4}{*}{ 11. Ignorar el problema (Ip) } & 12 & 2.0 & .42 * \\
\hline & 30 & 2.0 & .46 * \\
\hline & 48 & 2.3 & .33 * \\
\hline & 66 & 2.1 & .26 * \\
\hline \multirow[t]{4}{*}{ 12. Autoinculparse $(\mathrm{Cu})$} & 13 & 2.9 & .48 * \\
\hline & 31 & 2.9 & .32 * \\
\hline & 49 & 2.8 & $.57 *$ \\
\hline & 67 & 2.6 & .59 * \\
\hline
\end{tabular}

${ }^{*}{ }^{*}$ Ítem aceptado

$N=1236$

ítem 10 y .40 para el ítem 47, observándose que los ítems en su totalidad cumplieron con los criterios establecidos.

En la escala 11, Ignorar el problema, se puede ver que los valores promedio de los ítems se movieron entre 2.0 para los ítems 12 y 30 y 2.3 para el ítem 48 . En lo que se refiere a la correlación ítem-test corregido, ésta osciló entre
.26 para el ítem 66 y .46 para el ítem 30 , cumpliendo todos los ítems los criterios señalados.

En la escala 12, Autoinculparse, se puede observar que los valores promedio de los ítems variaron entre 2.6 para el ítem 67 y 2.9 para el ítem 13 y 31, mientras que los valores correspondientes a la correlación ítem-test corre- 
gido fluctuaron entre .32 para el ítem 31 y .59 para el ítem 67 , notándose que todos los ítems superaron los criterios establecidos.

En la escala 13, Reservarlo para sí, se puede ver que los valores promedio de los ítems fluctuaron entre 2.1 para el ítem 32 y 3.1 para el ítem 14. Respecto de la correlación ítem-test corregido, se observa que los valores oscilaron entre .25 para el ítem 32 y .50 para el ítem 68 , notándose que todos los ítems superaron los criterios establecidos.
En la escala 14, Buscar apoyo espiritual, se encuentra que los valores promedio de los ítems variaron entre 1.8 para el ítem 51 y 3.5 para el ítem 69. En lo que se refiere a la correlación ítem-test corregido, los valores se movieron entre .06 para el ítem 33 y .57 para el ítem 69 , observándose que de los cuatro ítems que conforman dicha escala sólo uno de ellos no superó los criterios establecidos; por lo tanto, debió ser eliminado. Éste fue el ítem 33: Pido ayuda y consejo para que se resuelvan mis problemas.

Tabla $N^{\circ} 3$

Análisis de ítems de la escala 13 a la escala 18

\begin{tabular}{|c|c|c|c|}
\hline Escalas & Item & M & ritc \\
\hline \multirow[t]{4}{*}{ 13. Reservarlo para sí (Re) } & 14 & 3.1 & $.40^{*}$ \\
\hline & 32 & 2.1 & .25 * \\
\hline & 50 & 3.0 & .42 * \\
\hline & 68 & 3.0 & $.50 *$ \\
\hline \multirow[t]{4}{*}{ 14. Buscar apoyo espiritual $(\mathrm{Ae})$} & 15 & 3.0 & $.53^{*}$ \\
\hline & 33 & 2.8 & .06 \\
\hline & 51 & 1.8 & .35 * \\
\hline & 69 & 3.5 & $.57^{*}$ \\
\hline \multirow[t]{4}{*}{ 15. Fijarse en lo positivo (Po) } & 16 & 2.8 & .22 * \\
\hline & 34 & 3.6 & .42 * \\
\hline & 52 & 3.6 & $.45^{*}$ \\
\hline & 70 & 2.8 & .23 * \\
\hline \multirow[t]{4}{*}{ 16. Buscar ayuda profesional (Ap) } & 17 & 3.1 & $.50 *$ \\
\hline & 35 & 1.8 & .62 * \\
\hline & 53 & 1.7 & .59 * \\
\hline & 71 & 2.7 & .56 * \\
\hline \multirow[t]{3}{*}{ 17. Buscar diversiones relajantes (Dr) } & 18 & 4.0 & .32 * \\
\hline & 36 & 3.1 & .28 * \\
\hline & 54 & 3.6 & .31 * \\
\hline \multirow[t]{3}{*}{ 18. Distracción física (Fi) } & 19 & 3.0 & $.65^{*}$ \\
\hline & 37 & 3.0 & $.70^{*}$ \\
\hline & 55 & 2.8 & $.77^{*}$ \\
\hline
\end{tabular}

$\left.{ }^{*}\right)$ Ítem aceptado

Nota: Todos los ítems fueron aceptados, excepto el 33.

$\mathrm{N}=1236$ 
La escala 15, Fijarse en lo positivo, presentó valores promedio de los ítems, que se movieron entre 2.8 para los ítems 16 y 70 y 3.6 para los ítems 34 y 70; mientras que en la correlación ítem-test corregido los valores fluctuaron entre .22 para el ítem 16 y .45 para el ítem 52, observándose que todos los ítems cumplieron con los criterios determinados.

En la escala 16, Buscar ayuda profesional, puede verse que los valores promedio de los ítems oscilaron entre 1.7 para el ítem 53 y 3.1 para el ítem 17. Con relación a la correlación ítem-test corregido, los valores obtenidos variaron entre .50 para el ítem 17 y .62 para el ítem 35, notándose que los ítems en su totalidad cumplieron con los criterios mencionados.

En la escala 17, Buscar diversiones relajantes, se encuentra que los valores promedio de los ítems se movieron entre 3.1 para el ítem 36 y 4.0 para el ítem 18 ; mientras que los valores correspondientes a la correlación ítem-test corregido fluctuaron entre .28 para el ítem 36 y .32 para el ítem 18 , encontrándose que todos los ítems pertenecientes a dicha escala superaron los criterios ya indicados.

Finalmente, en la escala 18, Distracción física, se puede observar que los valores promedios de los ítems oscilaron entre 2.8 para el ítem 55 y 3.0 para los ítems 19 y 37 . Respecto a la correlación ítem-test corregido, los valores variaron entre .65 para el ítem 19 y .77 para el ítem 55, notándose que todos los ítems cumplieron los dos criterios establecidos.

\section{Análisis factorial de los ítems del ACS Lima}

El análisis factorial realizado según el método de los componentes principales y la rotación oblicua oblimin fue llevado a cabo en tres etapas o muestras de ítems, como en la versión original, pues de esta forma se evitaron las dificultades estadísticas de analizar en un solo paso los 80 reactivos agrupados en 18 escalas. Cada una de las tres muestras agrupó aleatoriamente seis escalas o estrategias diferentes con sus ítems respectivos.

Los resultados del análisis factorial en las tres etapas mostraron que las medidas previas, como son el KaiserMeyer-Olkin de adecuación al muestreo y el test de esfericidad de Bartlett, indicaron la pertinencia de realizar el análisis estadístico, ya que es factible obtener resultados relevantes.

Seguidamente se presentan estos resultados en las tablas $\mathrm{N}^{\mathrm{o}} 4, \mathrm{~N}^{\mathrm{o}} 5 \mathrm{y} \mathrm{N}^{\mathrm{o}} 6$.

Como puede observarse en la tabla $\mathrm{N}^{\circ} 4$, el análisis factorial indicó la existencia de seis factores, los cuales permitieron explicar el $50,2 \%$ de la varianza total. El primer factor, que permitió explicar el 12,8\% de la varianza total, incluyó todos los ítems de la escala 15 Fijarse en lo positivo, además del ítem 17 Me preocupo por el futuro del mundo, perteneciente a la escala 4 
Tabla $N^{\circ} 4$

Análisis factorial solución oblimin de la muestra 1 de los ítems del ACS Lima

\begin{tabular}{|c|c|c|c|c|c|c|c|c|}
\hline \multirow[b]{2}{*}{ Ítems } & \multirow{2}{*}{\multicolumn{2}{|c|}{ Escala }} & \multicolumn{6}{|c|}{ Factor } \\
\hline & & & 1 & 2 & 3 & 4 & 5 & 6 \\
\hline 16 & 15 & Fijarse en lo positivo & .62 & & & & & \\
\hline 34 & 15 & Fijarse en lo positivo & .61 & & & & & \\
\hline 52 & 15 & Fijarse en lo positivo & .66 & & & & & \\
\hline 70 & 15 & Fijarse en lo positivo & .48 & & & & & \\
\hline 75 & 4 & Preocuparse & .63 & & & & & \\
\hline 13 & 12 & Autoinculparse & & .69 & & & & \\
\hline 31 & 12 & Autoinculparse & & .49 & & & & \\
\hline 49 & 12 & Autoinculparse & & .77 & & & & \\
\hline 67 & 12 & Autoinculparse & & .81 & & & & \\
\hline 66 & 11 & Ignorar el problema & & .49 & & & & \\
\hline 12 & & Ignorar el problema & & & .75 & & & \\
\hline 30 & & Ignorar el problema & & & .74 & & & \\
\hline 48 & & Ignorar el problema & & & .66 & & & \\
\hline 11 & & Acción social & & & & .55 & & \\
\hline 29 & & Acción social & & & & .64 & & \\
\hline 47 & & Acción social & & & & .75 & & \\
\hline 65 & 10 & Acción social & & & & .69 & & \\
\hline 19 & & Distracción física & & & & & -.83 & \\
\hline 37 & 18 & Distracción física & & & & & -.86 & \\
\hline 55 & & Distracción física & & & & & -.90 & \\
\hline 04 & & Preocuparse & & & & & & .44 \\
\hline 23 & & Preocuparse & & & & & & .66 \\
\hline 41 & 4 & Preocuparse & & & & & & .59 \\
\hline 59 & & Preocuparse & & & & & & .69 \\
\hline 10 & 10 & Acción social & & & & & & .53 \\
\hline Eigen & alue & & 3.2 & 2.7 & 2.4 & 1.7 & 1.6 & 1.0 \\
\hline $\begin{array}{l}\text { Propo } \\
\text { explica }\end{array}$ & $\begin{array}{l}\text { ión } \\
\text { la ac }\end{array}$ & $\begin{array}{l}\text { de varianza } \\
\text { cumulada }\end{array}$ & 12.8 & 23.6 & 33.1 & 39.8 & 46.1 & 50.2 \\
\hline
\end{tabular}

Kaiser-Meyer-Olkin. Medida de adecuación del muestreo $=.75$

Test de esfericidad de Bartlett $=6167.98^{*}$

$\left(^{*}\right)$ Significativo $p<.05$

$\mathrm{N}=1236$

Preocuparse. Esto estaría indicando que este primer factor pone énfasis en la escala 15, y que el ítem 75 guarda alguna relación con los demás ítems de dicha escala, relación que podría ser explicada por el hecho de que el preo- cuparse por lo que suceda en el futuro podría tener una connotación optimista y positiva frente a los problemas o preocupaciones que puedan presentarse más adelante. 
El segundo factor explicó el 10,8\% de la varianza total y agrupó todos los ítems de la escala 12 Autoinculparse y al ítem 66 de la escala 11 Ignorar el problema, que es Cuando tengo problemas, me aíslo para poder evitarlos. Por lo tanto, este factor estaría poniendo énfasis en la escala 12 y la inclusión del ítem 66 a este segundo factor podría deberse a que, en general, los ítems de la escala 12 indican una tendencia por parte del sujeto a verse como culpable y a criticarse frente a los problemas, lo cual podría provocar como consecuencia un aislamiento del problema.

El tercer factor, que explicó el 9,5\% de la varianza total, incluyó tres de los cuatro ítems de la escala 11 Ignorar el problema, lo cual indicaría que tal factor pone énfasis en la escala de Ignorar el problema. Esto mismo sucedió con el cuarto factor, que explicó el $6,7 \%$ de la varianza total y reunió a cuatro de los cinco ítems de la escala 10 Acción social, señalando igualmente que dicho factor estaría dando importancia a la mencionada escala. Asimismo, el quinto factor, que explicó el 6,3\% de la varianza total, incluyó los tres ítems de la escala 18 Distracción física, lo que significaría también que este factor le da énfasis a tal escala.

El sexto factor de esta primera muestra explicó el 4,1\% de la varianza total, reuniendo además a cuatro de los cinco ítems de la escala 4 Preocuparse y al ítem 10 (Organizo una acción en relación con mi problema) perteneciente a la escala 10 Acción social. Esto quiere decir que este factor estaría poniendo énfasis en la escala señalada y la inclusión del ítem 10 podría ser explicada como una respuesta puesta en marcha frente a las preocupaciones desencadenadas por el problema.

En la tabla $\mathrm{N}^{\circ} 5$ el segundo análisis factorial señaló igualmente la existencia de seis factores que han permitido explicar el 50,3\% de la varianza total. El primer factor de este segundo análisis explicó el $17,4 \%$ de la varianza total e incluyó todos los ítems de la escala 1 Buscar apoyo social más el ítem 33 de la escala 14 Buscar ayuda espiritual, que es Pido ayuda y consejo para que se resuelvan mis problemas. Esto estaría indicando que este primer factor pone énfasis en la escala de Buscar apoyo social y que, además, el hecho de que el ítem 33 se haya ubicado en este factor podría deberse a que guarda una estrecha relación semántica con los ítems de la primera escala, los cuales remarcan la búsqueda de ayuda para el afronte y la resolución de los problemas.

El segundo factor, que explicó el 9,1\% de la varianza total, reunió a cuatro de los cinco ítems de la escala 9 Reducción de la tensión; por lo tanto, este factor estaría poniendo énfasis en dicha escala. Igualmente, el tercer factor, que explicó el $8,5 \%$ de la varianza total, incluyó tres de los cuatro ítems de la escala 14 Buscar apoyo espiritual, lo cual indicaría también que dicho factor da importancia a la mencionada escala. 
Tabla $N^{\circ} 5$

Análisis factorial solución oblimin de la muestra 2 de los ítems del ACS Lima

\begin{tabular}{|c|c|c|c|c|c|c|c|}
\hline \multirow[b]{2}{*}{ Ítems } & \multirow[b]{2}{*}{ Escala } & \multicolumn{6}{|c|}{ Factor } \\
\hline & & 1 & 2 & 3 & 4 & 5 & 6 \\
\hline 01 & 1 Buscar apoyo social & .68 & & & & & \\
\hline 20 & 1 Buscar apoyo social & .71 & & & & & \\
\hline 38 & 1 Buscar apoyo social & .47 & & & & & \\
\hline 56 & 1 Buscar apoyo social & .83 & & & & & \\
\hline 72 & 1 Buscar apoyo social & .72 & & & & & \\
\hline 33 & 14 Buscar ayuda espiritual & .73 & & & & & \\
\hline 09 & 9 Reducción de la tensión & & .71 & & & & \\
\hline 28 & 9 Reducción de la tensión & & .56 & & & & \\
\hline 64 & 9 Reducción de la tensión & & .62 & & & & \\
\hline 80 & 9 Reducción de la tensión & & .80 & & & & \\
\hline 15 & 14 Buscar apoyo espiritual & & & .78 & & & \\
\hline 51 & 14 Buscar apoyo espiritual & & & .64 & & & \\
\hline 69 & 14 Buscar apoyo espiritual & & & .81 & & & \\
\hline 06 & 6 Buscar pertenencia & & & & .69 & & \\
\hline 25 & 6 Buscar pertenencia & & & & .67 & & \\
\hline 43 & 6 Buscar pertenencia & & & & .62 & & \\
\hline 61 & 6 Buscar pertenencia & & & & .75 & & \\
\hline 07 & 7 Hacerse ilusiones & & & & .52 & & \\
\hline 62 & 7 Hacerse ilusiones & & & & .42 & & \\
\hline 78 & 7 Hacerse ilusiones & & & & .45 & & \\
\hline 26 & 7 Hacerse ilusiones & & & & & .66 & \\
\hline 44 & 7 Hacerse ilusiones & & & & & .70 & \\
\hline 46 & 9 Reducción de la tensión & & & & & .60 & \\
\hline 77 & 6 Buscar pertenencia & & & & & .52 & \\
\hline 18 & 17 Buscar diversiones relajantes & & & & & & .70 \\
\hline 36 & 17 Buscar diversiones relajantes & & & & & & .65 \\
\hline 54 & 17 Buscar diversiones relajantes & & & & & & .60 \\
\hline \multicolumn{2}{|c|}{ Eigen value } & 4.7 & 2.5 & 2.3 & 1.7 & 1.3 & 1.1 \\
\hline \multicolumn{2}{|c|}{$\begin{array}{l}\text { Proporción de varianza } \\
\text { explicada acumulada }\end{array}$} & 17.4 & 26.5 & 35.0 & 41.4 & 46.1 & 50.3 \\
\hline
\end{tabular}

Kaiser-Meyer-Olkin Medida de adecuación del muestreo $=.82$

Test de esfericidad de Bartlett $=7540.81^{*}$

$\left({ }^{*}\right)$ Significativo $p<.05$

$\mathrm{N}=1236$

El cuarto factor explicó el 6,4\% de la varianza total y agrupó cuatro de los cinco ítems de la escala 6 Buscar pertenencia, siendo éstos: el ítem 6 Trato de dar una buena impresión en las perso- nas que me importan; el ítem $25 \mathrm{Me}$ preocupo por mis relaciones con los demás; el ítem 43 Trato de adaptarme a mis amigos; y el ítem 61 Trato de mejorar mi relación personal con los de- 
más. Se encontraron también tres ítems de la escala 7 Hacerse ilusiones, los cuales fueron: el ítem 7 Espero que me ocurra lo mejor; el ítem 62 Sueño despierto(a) que las cosas van a mejorar; y el ítem $78 \mathrm{Me}$ imagino que las cosas van a ir mejor. La inclusión de estos tres ítems de la escala 7 Hacerse ilusiones a la escala 6 Buscar pertenencia podría deberse a que el interés y la preocupación por mejorar la relación con los demás, sean amigos u otras personas significativas, y la posibilidad de tener relaciones personales positivas guardaría cierta relación con la esperanza de que los problemas tendrán una salida positiva.

El quinto factor, que explicó el 14,7\% de la varianza total, reunió dos ítems de la escala 7 Hacerse ilusiones, los cuales fueron: el ítem 26 Espero que un milagro resuelva mis problemas, y el ítem 44 Espero que el problema se resuelva por sí solo. Así como también el ítem 46 Culpo a los demás de mis problemas de la escala 9 Reducción de la tensión y el ítem 77 Hago lo que quieren mis amigos de la escala 6 Buscar pertenencia. Puede decirse, por un lado, que esta dimensión ha puesto énfasis en la escala 7 Hacerse ilusiones por la mayor presencia de ítems pertenecientes a esta escala y, por otro, que la agrupación de estos cuatro ítems en un factor tendría como explicación el hecho de que ellos tienen en común poner afuera la causa y el manejo de los problemas o las preocupaciones.
Por último, el sexto factor explicó el $4,2 \%$ de la varianza total e incluyó todos los ítems de la escala 17 Buscar diversiones relajantes, lo cual estaría indicando que este factor pone énfasis en la mencionada escala.

En la tabla $\mathrm{N}^{\mathrm{o}} 6$ se puede observar que el tercer análisis factorial señaló la existencia de seis factores, que explicaron el 49.7 de la varianza total. El primer factor, que explicó el 16.2 de la varianza total, reunió todos los ítems de la escala 2, Concentrarse en resolver el problema, lo cual estaría indicando que este factor le da énfasis a esta segunda escala. Sin embargo, se incluyeron también, de manera positiva, el ítem 58 Busco tener éxito en las cosas que estoy haciendo, de la escala 3 Esforzarse y tener éxito; y de manera negativa los ítems 8 Como no puedo hacer nada para resolver el problema, no haga nada y 27 Frente a los problemas, simplemente me doy por vencido(a) de la escala 8 Falta de afrontamiento o No afrontamiento. Esto estaría indicando que en este primer factor se complementan ítems que tienen que ver con el manejo directo y activo del problema y otros que se relacionan, por el contrario, con conductas de evasión frente al problema.

En el segundo factor, que explicó el $8,7 \%$ de la varianza total, podemos notar que se han agrupado los cuatro ítems de la escala 16 Buscar ayuda profesional, lo cual estaría reflejando que dicho factor pone énfasis en la mencionada escala. 
Tabla $N^{\circ} 6$

Análisis factorial solución oblimin de la muestra 3 de los ítems del ACS Lima

\begin{tabular}{|c|c|c|c|c|c|c|c|}
\hline \multirow[b]{2}{*}{ Ítems } & \multirow[b]{2}{*}{ Escala } & \multicolumn{5}{|c|}{ Factor } & \multirow[b]{2}{*}{6} \\
\hline & & 1 & 2 & 3 & 4 & 5 & \\
\hline 02 & 2 Concentrarse en resolver el problema & .70 & & & & & \\
\hline 21 & 2 Concentrarse en resolver el problema & .71 & & & & & \\
\hline 39 & 2 Concentrarse en resolver el problema & .50 & & & & & \\
\hline 57 & 2 Concentrarse en resolver el problema & .60 & & & & & \\
\hline 73 & 2 Concentrarse en resolver el problema & .70 & & & & & \\
\hline 58 & 3 Esforzarse y tener éxito & .54 & & & & & \\
\hline 08 & 8 Falta de afrontamiento o no afrontamiento & -.45 & & & & & \\
\hline 27 & 8 Falta de afrontamiento o no afrontamiento & -.47 & & & & & \\
\hline 17 & 16 Buscar ayuda profesional & & .62 & & & & \\
\hline 35 & 16 Buscar ayuda profesional & & .84 & & & & \\
\hline 53 & 16 Buscar ayuda profesional & & .81 & & & & \\
\hline 71 & 16 Buscar ayuda profesional & & .69 & & & & \\
\hline 14 & 13 Reservarlo para sí & & & .60 & & & \\
\hline 32 & 13 Reservarlo para sí & & & .46 & & & \\
\hline 50 & 13 Reservarlo para sí & & & .65 & & & \\
\hline 68 & 13 Reservarlo para sí & & & .70 & & & \\
\hline 63 & 8 Falta de afrontamiento o no afrontamiento & & & .51 & & & \\
\hline 05 & 5 Invertir en amigos íntimos & & & & .68 & & \\
\hline 24 & 5 Invertir en amigos íntimos & & & & .66 & & \\
\hline 42 & 5 Invertir en amigos íntimos & & & & .66 & & \\
\hline 60 & 5 Invertir en amigos íntimos & & & & .59 & & \\
\hline 76 & 5 Invertir en amigos íntimos & & & & .72 & & \\
\hline 03 & 3 Esforzarse y tener éxito & & & & & .71 & \\
\hline 40 & 3 Esforzarse y tener éxito & & & & & .61 & \\
\hline 74 & 3 Esforzarse y tener éxito & & & & & .74 & \\
\hline 22 & 3 Esforzarse y tener éxito & & & & & & .41 \\
\hline 45 & 8 Falta de afrontamiento o no afrontamiento & & & & & & -.77 \\
\hline 79 & 8 Falta de afrontamiento o no afrotnameinto & & & & & & -.78 \\
\hline$\overline{\text { Eigen }}$ & $n$ value & 4.5 & 2.4 & 2.3 & 1.9 & 1.5 & 1.3 \\
\hline Propo & orción de varianza explicada acumulada & 16.2 & 24.9 & 33.3 & 40.0 & 45.3 & 49.7 \\
\hline
\end{tabular}

Kaiser-Meyer-Olkin Medida de adecuación del muestreo $=.80$

Test de esfericidad de Bartlett $=8080.51^{*}$

$\left({ }^{*}\right)$ Significativo $p<.05$

$\mathrm{N}=1236$

El tercer factor, que explicó el 8,4\% de la varianza total, abarcó todos los ítems de la escala 13 Reservarlo para sí, y al ítem 63 Cuando tengo problemas, no sé como enfrentarlos de la es- cala 8 Falta de afrontamiento o No afrontamiento. Por lo tanto, este factor estaría indicando que se le da mayor importancia a la escala de Reservarlo para sí, y que además el ítem 63 perte- 
neciente a la escala Falta de afrontamiento o No afrontamiento se relaciona de alguna forma con los ítems de la escala Reservarlo para sí. Es interesante notar que los cinco ítems incluidos en este factor hacen referencia a una tendencia a la evasión, sea al problema mismo o al apoyo de los demás para afrontar y solucionar el problema.

El cuarto factor explicó el 6,7\% de la varianza total e incluyó solamente a los cinco ítems de la escala 5 Invertir en amigos íntimos, lo cual estaría indicando que este factor pone énfasis en dicha escala. Así mismo, el quinto factor, que explicó el 5,3\% de la varianza total, reunió a tres de los cinco ítems de la escala 3 Esforzarse y tener éxito; por lo tanto, dicho factor estaría mostrando un énfasis mayor en la escala 3.

El sexto factor, que explicó el 4.4 de la varianza total, incluyó de manera positiva al ítem 22 Sigo asistiendo a clases de la escala 3 Esforzarse y tener éxito, y de manera negativa, a los ítems 45 Me pongo mal (Me enfermo) y 79 Sufro dolores de cabeza o de estómago de la escala 8 Falta de afrontamiento o No afrontamiento, indicando esto último que este sexto factor pone énfasis en la escala 8 . Puede notarse también que, al igual que en el primer factor de este tercer análisis factorial, en este factor se agrupan en forma complementaria el afrontamiento activo de los problemas junto con conductas que reflejan más bien dificultades para enfrentarse a éstos, como son el desarrollo de síntomas psicosomáticos.
Finalmente, puede concluirse que los resultados de los análisis factoriales realizados por partes revelaron que se mantienen las escalas con la inclusión en algunos casos de ítems específicos, lo cual nos indicaría que la prueba tendría validez de constructo.

\section{Análisis factorial de las escalas del ACS Lima}

En la tabla $\mathrm{N}^{\circ} 7$ puede observarse que a partir del análisis factorial de las escalas del ACS revisado se identificó la existencia de seis factores, los cuales permitieron explicar el $67,6 \%$ de la varianza total. El primer factor, que explicó el $25 \%$ de la varianza total, estuvo conformado por las siguientes escalas: Concentrarse en resolver el problema (.76), Esforzarse y tener éxito (.71) y Preocuparse (.77), las cuales aluden a diferentes formas de enfrentar el problema. Por lo tanto, esta dimensión podría recibir el nombre de Afrontamiento activo frente al problema.

El segundo factor, que explicó el $14,7 \%$ de la varianza total, estuvo compuesto por tres escalas: Falta de afrontamiento o No afrontamiento (.71), Reducción de la tensión (.78) y Autoinculparse (.72), las cuales se relacionan con una incapacidad del sujeto para afrontar los problemas, razón por la cual podrían agruparse bajo la dimensión denominada Afrontamiento no productivo.

El tercer factor explicó el 9,5\% de la varianza total y estuvo conformado por 
Tabla $N^{0} 7$

Análisis factorial solución oblimin de las escalas de afrontamiento del ACS Lima

\begin{tabular}{|c|c|c|c|c|c|c|c|}
\hline & & \multicolumn{6}{|c|}{ Factor } \\
\hline & & 1 & 2 & 3 & 4 & 5 & 6 \\
\hline \multicolumn{8}{|c|}{ Escalas } \\
\hline E2: & Concentrarse en resolver el problema (Rp) & .76 & & & & & \\
\hline E3: & Esforzarse y tener éxito (Es) & .71 & & & & & \\
\hline E4: & Preocuparse (Pr) & .77 & & & & & \\
\hline E8: & Falta de afrontamiento o no afrontamiento (NA) & & .71 & & & & \\
\hline E9: & Reducción de la tensión (Rt) & & .78 & & & & \\
\hline E12: & Autoinculparse $(\mathrm{Cu})$ & & .72 & & & & \\
\hline E11: & Ignorar el problema (Ip) & & & .57 & & & \\
\hline E13: & Reservarlo para sí (Re) & & & .68 & & & \\
\hline E5: & Invertir en amigos íntimos (Ai) & & & & .64 & & \\
\hline E6: & Buscar pertenencia $(\mathrm{Pe})$ & & & & .54 & & \\
\hline E17: & Buscar diversiones relajantes (Dr) & & & & .79 & & \\
\hline E18: & Distracción física (Fi) & & & & .70 & & \\
\hline E7: & Hacerse ilusiones $(\mathrm{Hi})$ & & & & & .68 & \\
\hline E14: & Buscar ayuda espiritual (Ae) & & & & & .80 & \\
\hline E15: & Fijarse en lo positivo (Po) & & & & & .56 & \\
\hline E1: & Buscar apoyo social (As) & & & & & & .76 \\
\hline E10: & Acción social (So) & & & & & & .81 \\
\hline E16: & Buscar ayuda profesional (Ap) & & & & & & .84 \\
\hline$\overline{\text { Eigen }}$ & $\mathrm{n}$ value & 4.5 & 2.6 & 1.7 & 1.3 & 1.1 & .90 \\
\hline Propo & orción de varianza explicada acumulada & 25.0 & 39.7 & 49.2 & 56.4 & 62.6 & 67.6 \\
\hline
\end{tabular}

Kaiser-Meyer-Olkin Medida de adecuación del muestreo $=.82$

Test de esfericidad de Bartlett $=7127.24^{*}$

$\left({ }^{*}\right)$ Significativo $p<.05$

$\mathrm{N}=1236$

dos escalas: Ignorar el problema (.57) y Reservarlo para sí (.68). Ambas se refieren a un intento de negar el problema y aislarse de él y de las demás personas para tratar de evitarlo; por lo tanto, esta dimensión podría llamarse Distanciamiento en general.

El cuarto factor, que explicó el 7,2\% de la varianza total, reunió las siguientes escalas: Invertir en amigos íntimos (.64), Buscar pertenencia (.54), Buscar diversiones relajantes (.79) y Distrac- ción física (.70). Estas escalas aluden a la búsqueda de personas significativas, como, por ejemplo, amigos o sujetos del sexo opuesto, con el fin de realizar actividades relajantes y físicas. Por lo tanto, podría decirse que forman una única dimensión a la que se denominaría Búsqueda de apoyo social y emocional frente al problema.

El quinto factor, que explicó el 6,2\%, incluyó tres escalas: Hacerse ilusiones (.68), Buscar ayuda espiritual (.80) y Fi- 
jarse en lo positivo (.56). Éstas tienen que ver con el hecho de afrontar los problemas, reflejando una visión positiva y optimista y teniendo expectativas de que éstos se podrán resolver; por lo tanto, esta dimensión podría llamarse Interpretación positiva del problema.

Finalmente, el sexto factor, que explicó el 5,0\% de la varianza total, reunió a las siguientes escalas: Buscar apoyo social (.76), Acción social (.81) y Buscar ayuda profesional (.84), que hacen referencia a la búsqueda de ayuda y opinión de los demás, sean personas cercanas o profesionales, para afrontar y resolver el problema. Por esta razón, dicha dimensión podría recibir el nombre de Búsqueda de apoyo social instrumental frente al problema.

\section{Análisis de la confiabilidad por consistencia interna de las escalas del ACS Lima}

En la tabla $\mathrm{N}^{\circ} 8$ se puede observar que las escalas con los valores alfa más pequeños fueron la escala 17 Buscar diversiones relajantes con .48 y la escala 7 Hacerse ilusiones con .52; mientras que los valores alfa más altos correspondieron a las escalas 18 Distracción física con .84 y la escala 16 Buscar ayuda profesional con .76.

Puede apreciarse también que entre las escalas con valores alfa que fluctuaron entre .53 y .58 estuvieron las escalas 10 Acción social y 15 Fijarse en lo positivo, ambas con un valor alfa de .53 ; las escalas 3 Esforzarse y tener éxito y 8 Falta de afrontamiento o No afrontamiento, que alcanzaron un valor alfa de .56; y las escalas 11 Ignorar el problema y 14 Buscar apoyo espiritual, con un valor alfa .58. Asimismo, las escalas que presentaron valores alfa que oscilaron entre .61 y .66 son: las escalas 4 Preocuparse y 13 Reservarlo para sí, que alcanzaron un valor de .61; la escala 9 Reducción de la tensión con .63; y la escala 6 Buscar pertenencia, que presentó un valor de .66. Además, hubo escalas cuyos valores alfa se movieron entre los valores alfa .70 y .74. Estas escalas fueron las siguientes: la escala 12 Autoinculparse con un valor de .70; la escala 5 Invertir en amigos íntimos, que alcanzó un valor de .71; la escala 1 Buscar apoyo social, que obtuvo un valor de .73; y la escala 2 Concentrarse en resolver el problema con un valor de .74.

Por otra parte, se puede ver que el contraste estadístico de la significación de cada coeficiente alfa nos indicó que todos los resultados fueron significativos a una probabilidad mayor de .05 , por lo cual puede concluirse que todas las escalas del ACS Lima son confiables.

Una vez finalizado el proceso de validación y determinación de la consistencia interna del ACS Lima, se elaboraron los baremos en función del rango percentilar, tanto para el grupo de los varones como para el grupo de las mujeres. Éstos son presentados en el anexo 2. 
Tabla $N^{\circ} 8$

Confiabilidad por consistencia interna de las escalas del ACS Lima

\begin{tabular}{llcc}
\hline \multicolumn{1}{c}{ Escalas } & Alfa de Cronbach & $\mathrm{F}$ \\
\hline 1. Buscar apoyo social (As) & .73 & $4.17^{*}$ \\
2. Concentrarse en resolver el problema (Rp) & .74 & $3.85^{*}$ \\
3. Esforzarse y tener éxito (Es) & .56 & $2.27^{*}$ \\
4. Preocuparse (Pr) & .61 & 2.56 * \\
5. Invertir en amigos íntimos (Ai) & .71 & $3.45^{*}$ \\
6. Buscar pertenencia (Pe) & .66 & 2.94 * \\
7. Hacerse ilusiones (Hi) & .52 & 2.08 * \\
8. Falta de afrontamiento o No afrontamiento (Na) & .56 & $2.27^{*}$ \\
9. Reducción de la tensión (Rt) & .63 & 2.70 * \\
10. Acción social (So) & .53 & 2.13 * \\
11. Ignorar el problema (Ip) & .58 & 2.38 * \\
12. Autoinculparse (Cu) & .70 & 3.33 * \\
13. Reservarlo para sí (Re) & .61 & 2.56 * \\
14. Buscar apoyo espiritual (Ae) & .58 & 2.38 * \\
15. Fijarse en lo positivo (Po) & .53 & 2.13 * \\
16. Buscar ayuda profesional (Ap) & .76 & 4.17 * \\
17. Buscar diversiones relajantes (Dr) & .48 & 1.92 * \\
18. Distracción física (Fi) & .84 & $6.25^{*}$ \\
\hline
\end{tabular}

(*) Significativo $p<.05$

$\mathrm{GL} 1=1236$

GL2 = 3708

$\mathrm{N}=1236$

\section{DisCUSIÓN}

Los resultados del análisis psicométrico demuestran que la prueba Escalas de Afrontamiento para Adolescentes Revisadas (ACS Lima) alcanza niveles de validez y confiabilidad aceptables, de lo cual puede concluirse que dicha prueba puede funcionar en nuestro medio y es consistente con la versión española, a pesar de las diferencias culturales existentes entre los adolescentes españoles y los pertenecientes a nuestro medio.

Lo anterior es confirmado por los resultados obtenidos en el análisis de ítems de cada una de las escalas, a tra- vés del cual encontramos que, en general, todos los ítems de las 18 escalas, excepto uno, fueron aceptados y concordantes entre sí, lo cual demuestra que la versión modificada del ACS es consistente con la versión española.

Sólo uno de los 80 ítems no fue aceptado, pues la correlación ítem-test corregido, no fue mayor de 0.20. Este ítem fue el 33, Pido ayuda y consejo para que todo se resuelva, perteneciente a la escala 14, Buscar apoyo espiritual, que agrupa ítems que reflejan una tendencia a creer en la ayuda de Dios, rezando y empleando la oración. Es probable que la eliminación de dicho ítem se deba a que, a diferencia de los otros tres ítems 
que conforman la escala, éste no hace referencia explícita al hecho de acudir a la religión frente a los problemas, lo cual puede haber influido en que los escolares evaluados no lo consideren como perteneciente a la estrategia de búsqueda de ayuda espiritual. Esto estaría indicando, además, de acuerdo con $\mathrm{Mu}$ ñiz (1996a), que el ítem 33 no está conectado directamente con la variable que intenta medir, lo que hace que disminuya su índice de validez y que, por lo tanto, deba ser descartado.

Con respecto a la confiabilidad del ACS Lima, se encontró que, aunque los valores alfa alcanzados en la versión modificada del ACS fueron en su mayoría menores que los obtenidos en el Adolescent Coping Scale (ACS) de Frydenberg y Lewis, no dejaron de ser estadísticamente significativos, lo cual evidencia que la versión modificada de la prueba Escala de Afrontamiento para Adolescentes (ACS Lima) es confiable. Es posible que los puntajes menores obtenidos en el ACS Lima hayan tenido como factores principales el tamaño de la muestra del presente estudio, el cual fue mayor que la utilizada en la versión australiana, así como una menor variabilidad en la muestra limeña, lo que no permitiría el aumento de los coeficientes de confiabilidad (Anastasi y Urbina, 1998).

Los coeficientes de confiabilidad de las escalas fluctuaron en un rango que va de .48 para la escala de Buscar diversiones relajantes a .84 para la escala de Distracción física. El bajo coeficiente presentado por la escala de Buscar diversiones relajantes, que incluye actividades de ocio y relajantes, como oír música, ver la televisión, salir a divertirse con los amigos, etc., podría tener que ver con la poca variabilidad de los ítems de esta escala. Esto quiere decir que es propio en los adolescentes entre 14 y 17 años, pertenecientes a la muestra, la tendencia a realizar este tipo de actividades frente a los problemas, 10 que podría considerarse un elemento natural del desarrollo adolescente. Al respecto, Papalia (1990) señala que los adolescentes, en general, prefieren pasar su tiempo libre junto a sus amigos realizando cualquier actividad que les permita divertirse y distraerse de sus deberes, y evitan estar en casa con los padres, en donde las actividades tienden a ser más serias y monótonas.

Otro factor que se debe tener en cuenta, que podría explicar el coeficiente de confiabilidad alcanzado por la escala de Buscar diversiones relajantes, es el número de ítems que conforman esta escala, que es sólo de tres. Muñiz (1996b) plantea que cuantos más ítems se incluyan más aspectos del rasgo se podrán evaluar, lo que redundará en una mayor precisión métrica $\mathrm{y}$, por lo tanto, en un mayor coeficiente de confiabilidad.

Por el contrario, la escala de Distracción física alcanzó un coeficiente de confiabilidad alto de .84, lo cual nos estaría indicando una mayor variabili- 
dad y heterogeneidad en las respuestas dadas a los ítems de tal escala. Esto significa que no necesariamente todos los adolescentes incluidos en la muestra tienden a acudir de igual manera al deporte y al ejercicio físico como una forma de enfrentar sus problemas, pues, a diferencia de la estrategia que implica realizar actividades distractoras, la actividad física requiere de un mayor esfuerzo y de cierta disciplina para llevarla a cabo.

Asimismo, los ítems de la escala de Buscar ayuda profesional, que sigue a Distracción física, con un coeficiente de .76 , generan respuestas consistentes por parte de los estudiantes, lo cual estaría indicando que no todos ellos buscan la ayuda y el consejo de profesionales, y esto podría atribuirse básicamente a las características propias de esta etapa adolescente, en la que se busca mayormente el apoyo del grupo de amigos, más que de los padres u otros adultos, sean profesionales o no (Freyre, 1994). Además, la variabilidad en dicha escala podría deberse también a nuestra realidad, la cual no permite que los adolescentes de los diferentes grupos socioeconómicos accedan por igual a los servicios dados por profesionales.

Luego de realizar el análisis de ítems y de la confiabilidad, se llevó a cabo el análisis factorial tanto de las escalas del ACS Lima, como de los ítems. Los resultados hallados en ambos análisis confirman lo señalado por Hambleton (1996), quien plantea que las diferen- cias culturales, sociales, políticas, económicas y de idioma son un factor importante en la adaptación de los tests, afectando éstas tanto el significado y la interpretación de las puntuaciones y los procedimientos técnicos empleados, como el análisis factorial. Debe tenerse en cuenta, pues, este factor contextual, ya que el análisis factorial estará siempre afectado por las características socioculturales de la muestra que es utilizada. Si este factor no es atendido adecuadamente, puede obtenerse un test adaptado que no es equivalente en los idiomas o grupos culturales tomados en cuenta, conduciendo necesariamente a errores en la interpretación y a conclusiones defectuosas sobre los grupos estudiados (Muñiz, 1996b).

El análisis factorial de las escalas del ACS Lima, similar al realizado en la adaptación española, permitió definir seis dimensiones importantes.

En primer lugar, está la dimensión conformada por las escalas Concentrarse en resolver el problema, Esforzarse y tener éxito y Preocuparse, en donde los ítems pertenecientes a las dos primeras escalas se caracterizan por acciones que se dirigen a tratar el problema de manera sistemática, mostrando compromiso y dedicación, mientras que la escala de Preocuparse tiene que ver con el temor por el futuro (por las cosas que puedan pasar) y por la felicidad futura. Esto hace pensar que, aunque los escolares de la muestra estudiada tienden a preocuparse cuan- 
do experimentan preocupaciones o dificultades, esto no quiere decir que adopten actitudes pasivas o disfuncionales, sino más bien conductas que implican un manejo directo y responsable de los problemas, como si tales conductas fueran una consecuencia adaptativa de tales preocupaciones y temores. Es por esta razón que a dicha dimensión se le denomina Afrontamiento activo frente al problema, pues a pesar de la preocupación ligada a las dificultades, el manejo de ellas se caracteriza por ser productivo, analizando las causas y posibles soluciones, sin dejar de lado las actividades y obligaciones propias de la edad.

En segundo lugar, está la dimensión que agrupa a las escalas Falta de afrontamiento o No afrontamiento, Reducción de la tensión y Autoinculparse, caracterizadas más bien por un afrontamiento poco adaptativo y efectivo. Estas tres escalas guardarían, pues, cierta similitud, ya que se relacionan con un pobre rendimiento por parte del adolescente, quien o no puede hacer nada frente al problema, pudiendo desarrollar síntomas pisocosomáticos, o realiza acciones que permitan un alivio inmediato o, por último, se estanca en la autocrítica y en la culpa, sin llevar a cabo alguna acción. Debido, entonces, a la relación entre estas tres escalas, dicha dimensión es llamada Afrontamiento no productivo.
En tercer lugar, se encuentra la dimensión formada por las escalas Ignorar el problema y Reservarlo para sí, lo cual haría suponer que para los adolescentes la estrategia de Ignorar el problema, que implica un esfuerzo consciente por negar el problema o desentenderse de él, y la estrategia de Reservarlo para sí, que hace referencia a la tendencia del sujeto a alejarse de los demás para impedir que sepan cómo se siente uno frente a los problemas y cuáles son éstos, tendrían en común el hecho de tomar distancia, sea negando el problema o aislándose de los demás, razón por la cual se podría llamar a esta dimensión Distanciamiento en general. Además, la asociación entre ambas escalas podría significar que si se niega el problema o la preocupación se estaría evitando también que los demás sepan lo que sucede y cómo uno se siente, negándose no solamente el problema, sino el apoyo que los demás podrían dar.

Así mismo, se observa que la cuarta dimensión reúne las escalas Invertir en amigos íntimos, Buscar pertenencia, Buscar diversiones relajantes y Distracción física. Por un lado, se puede ver que la agrupación de la estrategia de Invertir en amigos íntimos, que se refiere al esfuerzo por buscar y comprometerse en relaciones íntimas, y la estrategia Buscar pertenencia, que implica la preocupación y el interés por parte del sujeto por sus relaciones con los demás y por lo que piensan de uno, podría deberse a la importancia que el 
grupo de los pares tiene en esta fase de la adolescencia, en la que también empieza a aparecer el interés por las relaciones heterosexuales (Fierro, 1986).

Pero, además, la unión de las escalas Buscar diversiones relajantes (que describe actividades de ocio como oír música, ver televisión, salir con los amigos, etc.), y Distracción física (que implica dedicarse al deporte y a los ejercicios físicos) con las dos primeras, hace pensar que los adolescentes vinculan necesariamente la realización de tales actividades con el grupo de amigos como, por ejemplo, ver una película o jugar un partido de fútbol, convirtiéndose éste en una importante red de soporte social, tendiendo los adolescentes a buscar apoyo básicamente emocional en ellos. Es por esto que a esta cuarta dimensión se le denomina Búsqueda de apoyo social y emocional frente al problema, ya que lo que se busca es el soporte moral, la simpatía y la comprensión por parte de los otros.

La quinta dimensión está constituida por las escalas Hacerse ilusiones, Buscar ayuda espiritual y Fijarse en lo positivo, las cuales tiene en común el hecho de guardar la esperanza de que se resolverán los problemas y tener la expectativa de que se recibirá algún apoyo, sea un milagro o la ayuda de Dios, para que todo tenga un final positivo y feliz, razón por la cual tal dimensión recibe el nombre de Interpretación positiva del problema. Es interesante notar que también podría pensarse que existe una tendencia a asociar lo religioso y espiritual con sentimientos basados en la esperanza y en el optimismo.

Finalmente, la sexta dimensión, conformada por las escalas Buscar apoyo social, Acción social y Buscar ayuda profesional, podría deberse a una similitud en el tipo de ítems incluidos en estas escalas, los cuales describen conductas orientadas a buscar un apoyo social más basado en lo instrumental que en lo emocional, es decir, en la búsqueda de información, consejo o asistencia. Por lo tanto, se ha denominado a esta última dimensión Búsqueda de apoyo social instrumental frente al problema.

Si se comparan estos resultados con la adaptación española del Adolescent Coping Scale, se observa que, aunque en el análisis factorial de las escalas sólo fueron definidas cuatro dimensiones, éstas guardan cierta similitud con las encontradas en la presente investigación. Tal parecido entre ambos análisis con adolescentes de diferentes culturas hace pensar que es probable que la prueba adaptada tenga cierta consistencia a nivel cultural. No obstante, debe reconocerse, a pesar de las diferencias culturales inherentes, la presencia de un mayor acercamiento cultural entre los adolescentes limeños y españoles, que aquel que se da entre los adolescentes limeños y los australianos.

Con respecto al análisis factorial de los ítems, es importante mencionar que 
en la adaptación española no se ha llevado a cabo este análisis, razón por la cual se comparan los resultados obtenidos en el presente estudio con los hallados en los análisis factoriales realizados por Frydenberg y Lewis (1996a).

En el primer análisis factorial se trabajaron las escalas: Preocuparse, Acción social, Ignorar el problema, Autoinculparse, Fijarse en lo positivo y Distracción física, y se encontró que solamente uno de los seis factores, el quinto factor, incluyó de manera pura la escala de Distracción física; notando también que dos factores, el tercer y cuarto factor, abarcaron, aunque de manera incompleta, las escalas Ignorar el problema y Acción social, respectivamente. En los factores restantes predominaron los ítems de una determinada escala, añadiéndose un ítem de otra escala diferente.

De este primer análisis, es importante destacar que el primer factor incluyó a todos los ítems de la escala Fijarse en lo positivo (que tiene que ver con una visión optimista y positiva de la situación presente y una tendencia a ver el lado bueno de las cosas) y al ítem 17 Me preocupo por el futuro del mundo perteneciente a la escala Preocuparse. La inclusión de este ítem en la escala de Fijarse en lo positivo, que, a diferencia de los otros cuatro ítems de la escala Preocuparse, no hace referencia a una preocupación con relación a uno mismo, podría significar que los adolescentes tienden mayormente a adop- tar una actitud positiva y optimista frente al futuro del mundo o incluso del país, que frente a problemas que tienen que ver con ellos mismos.

De manera similar, en el segundo factor predominaron todos los ítems de la escala Autoinculparse, agregándose el ítem 66 Cuando tengo problemas, me aíslo para poder evitarlos de la escala llamada Ignorar el problema. Tal inclusión podría ser entendida como una conducta puesta en marcha frente a la tendencia del sujeto a verse como culpable o responsable de sus dificultades, adoptando una actitud de crítica frente a los problemas. Esto hace pensar que dicha tendencia en los adolescentes a autoinculparse cuando experimentan preocupaciones tendría como consecuencia un intento por desentenderse del problema y evitarlo, lo cual se refleja en el aislamiento del problema descrito por el mencionado ítem.

Finalmente, en el sexto factor, que agrupó a cuatro de los cinco ítems de la escala Preocuparse y al ítem 10 Organizo una acción en relación con mi problema, perteneciente a la escala de Acción social, se encontró también que la presencia de este ítem puede entenderse, al igual que en el segundo factor, como una acción puesta en marcha frente a la tendencia a preocuparse por los problemas. Además, esto podría significar que en los adolescentes la estrategia de Preocuparse, aunque refleja cierta pasividad e improductividad, está asociada a su vez a la adopción de al- 
guna actividad, por lo que puede concluirse que, frente a las dificultades, los adolescentes manifiestan no solamente actitudes pasivas, sino que también tienden a la acción.

En el segundo análisis factorial, que incluyó a las siguientes escalas o estrategias: Buscar apoyo social, Buscar apoyo espiritual, Buscar diversiones relajantes, Buscar pertenencia, Hacerse ilusiones y Reducción de la tensión, se observó que los ítems no se agruparon de la misma manera que en la versión australiana del ACS. Sólo el sexto factor que agrupó a los tres únicos ítems de la escala Buscar diversiones relajantes guardó similitud con el análisis factorial del ACS original.

El primer factor de este segundo análisis factorial comprendió a los cinco ítems de la escala Buscar apoyo social más el ítem 33 Pido ayuda y consejo para que todo se resuelva, perteneciente a la escala Buscar apoyo espiritual. Es importante resaltar en este punto que la inclusión del este ítem en la escala de Buscar apoyo social podría deberse a una estrecha similitud semántica con los ítems de dicha escala, los cuales hacen referencia a una inclinación a compartir el problema con los otros, a conseguir ayuda para enfrentarlo y a buscar ayuda para encontrar una solución. El ítem 33, en cambio, no enfatiza como los demás reactivos de la estrategia Buscar ayuda espiritual, la orientación hacia Dios y la espera de su apoyo para la resolución del problema.
Además de lo anterior, podría pensarse también que dicha inclusión se debe a que las escalas Buscar apoyo social y Buscar apoyo espiritual, forman parte de un mismo estilo denominado Referencia a otros, el cual implica un intento por afrontar el problema acudiendo al apoyo y a los recursos de las demás personas (Frydenberg y Lewis, 1993a; 1997a).

Asímismo, puede observarse que el cuarto factor de este segundo análisis reunió a cuatro de los cinco ítems de la escala Buscar pertenencia y a tres ítems de la escala Hacerse ilusiones. La relación entre los ítems de ambas escalas podría residir en el hecho de que el interés y la preocupación del sujeto por establecer y mantener relaciones personales positivas, sean con los amigos $\mathrm{u}$ otras personas significativas, tratando de causar una buena impresión en ellos y prestando además atención a lo que ellos puedan pensar, tendría que ver con la tendencia a esperar que la situación va a mejorar y va a tener una salida positiva. Esto significaría que los adolescentes incluidos dentro de la muestra consideran la pertenencia a un grupo de amigos y la calidad de sus relaciones como un factor importante e influyente en la anticipación de una salida positiva frente a los problemas.

Finalmente, se encontró que el quinto factor agrupó a dos ítems de la escala Hacerse ilusiones (los ítems 26 Espero que un milagro resuelva mis problemas y 44 Espero que el problema se resuel- 
va por sí solo), al ítem 46 Culpo a los demás de mis problemas, de la escala de Reducción de la tensión y al ítem 77 Hago lo que quieren mis amigos, perteneciente a la escala Buscar pertenencia. Estos cuatros ítems reunidos en dicho factor podrían tener en común el hecho de que colocan la causa y el manejo del problema en el entorno, lo cual podría estar indicando que algunos adolescentes tienden a afrontar las dificultades, adoptando cierto locus de control externo, así como también cierta actitud pasiva.

En el tercer análisis factorial las escalas seleccionadas fueron: Concentrarse en resolver el problema, Buscar ayuda profesional, Invertir en amigos íntimos, Esforzarse y tener éxito, Falta de afrontamiento o No afrontamiento y Reservarlo para sí. En general, en dicho análisis observamos que los ítems de las escalas Esforzarse y tener éxito y Falta de afrontamiento o No afrontamiento, aparecieron dispersos dentro de los seis factores, mientras que los ítems de las escalas restantes adquirieron un predominio en los factores resultantes.

Así, en el primer factor se agruparon todos los ítems de la escala Concentrarse en resolver el problema, incluyéndose además el ítem 58 Busco tener éxito en las cosas que estoy haciendo, perteneciente a la escala de Esforzarse y tener éxito, y de manera negativa los ítems 8 Como no puedo hacer nada para resolver el problema, no hago nada y
27 Frente a los problemas, simplemente me doy por vencido(a), de la escala Falta de afrontamiento o No afrontamiento. Respecto de esto, podemos inferir, en primer lugar, que los adolescentes de nuestro medio tienden a asociar el abordaje directo y sistemático de los problemas, teniendo en cuenta las diferentes opciones de solución, con la obtención del logro gracias al esfuerzo realizado. Esto quiere decir, entonces, que al afrontar de manera directa los problemas, los adolescentes esperan tener como resultado el éxito en las acciones puestas en marcha, lo cual parece quedar confirmado por la existencia de cierta tendencia a no considerar las conductas de evasión frente al problema como una posibilidad para el afrontamiento.

De igual manera, se presentó este mismo patrón en el sexto factor, que incluyó de manera positiva el ítem 22 Sigo asistiendo a clases, de la escala Esforzarse y tener éxito y, de manera negativa, a dos ítems de la escala Falta de afrontamiento o No afrontamiento: el ítem 45 Me pongo mal (Me enfermo) y el ítem 79 Sufro dolores de cabeza o de estómago. En este factor se encontró, pues, una relación opuesta entre un afrontamiento directo y un afrontamiento evitativo, pudiendo inferir que en los escolares seleccionados podría haber una tendencia a relacionar en forma inversa la asistencia permanente a clases, aunque se experimenten problemas, con el desarrollo de síntomas psicosomáti- 
cos. Esto significaría, entonces, que se puede, por un lado, continuar con las responsabilidades propias de esta edad como es la escuela o, por el contrario, se reflejan dificultades para hacer frente a los problemas con la manifestación de somatizaciones.

Asimismo, en el tercer factor observamos que el ítem 63 Cuando tengo problemas, no sé cómo enfrentarlos, correspondiente a la escala Falta de afrontamiento o No afrontamiento se agrupó con todos los ítems de la escala Reservarlo para sí, lo cual hace pensar que la tendencia a huir de los demás para evitar que se enteren de los problemas trae como consecuencia que se experimente cierta incapacidad para afrontarlos. En este sentido, podría considerarse que es justamente este aislamiento de los otros, lo que probablemente llevaría al adolescente a sentirse incapaz de enfrentar el problema, ya que no se permite el apoyo de las personas que lo rodean.

En conclusión, en los resultados de los tres análisis factoriales de la versión modificada del ACS se encuentra que las escalas se mantienen, aunque se incluyan en algunas de ellas algunos ítems que en la prueba original no lo estaban. Probablemente esto se deba a las diferencias culturales entre los adolescentes australianos y los adolescentes de nuestro medio, las cuales afectan la estructura factorial de la prueba, debiéndose prestar también atención a los efectos de las traducciones hechas a ella, pues debido a éstas ciertas finuras de significado pueden haberse perdido. Sin embargo, a pesar de ello, puede decirse que los hallazgos obtenidos permiten concluir que el ACS Lima posee validez de constructo. 


\section{REFERENCIAS}

Anastasy, A. \& Urbina, S. (1998). Test psicológicos. México DF: Prentice Hall 7a. Ed.

Apoyo Opinión y Mercado, S.A. (1999). Niveles socioeconómicos en Lima metropolitana. Lima.

Ayers, T; Sandler, I. \& Twohey, J. (1998). Conceptualization and measurement of coping in children and adolescents. En: Ollendick, T. \& Prinz, R. (Eds). Advances in clinical psychology, 20, 243-301. New York: Plenum Press.

Boekaerts, M. (1996). Coping with stress in childhood and adolescence. En: Zeidner, M. \& Endler, N. (Eds.), Handbook of coping. Theory, research and applications, 452-484. New York: John Wiley.

Cedro (1999). Epidemiología de drogas en la población urbana peruana, 1997. Lima: Cedro.

De Ridder, D. (1997). What is wrong with coping assessment? A review of conceptual and methodological issues. Psychology and Health, 12, 417-431.

Escurra, L. (1988). Cuantificación de la validez de contenido por criterio de jueces. Revista de Psicología, 6 (1-2), 103111.

Fierro, A. (1986). Desarrollo social y de la personalidad en la adolescencia. En: Carretero, M.; Palacios, J. \& Marchesi, A. (Eds.), Psicología evolutiva 3. Adolescencia, madurez y senectud, 95-138. Madrid: Alianza.

Freyre, E. (1994). La salud del adolescente. Aspectos médicos y psicosociales. Lima: Concytec.
Frydenberg, E. \& Lewis, R. (1991). Adolescent coping: The different ways in which boys and girls cope. Journal of Adolescence, 14, 119-133.

Frydenberg, E. \& Lewis, R. (1993a). Boys play sport and girls turn to others: age, gender and ethnicity as determinants of coping. Journal of Adolescence, 16, 253-266.

Frydenberg, E. (1993b). The coping strategies used by capable adolescents. Australian Journal of Guidance \& Counselling, 3 (1), 1-9.

Frydenberg, E. \& Lewis, R. (1994). Coping with different concerns: Consistency and variation in coping strategies used by adolescents. Australian Psychologist, 29 (1), 45-48.

Frydenberg, E. \& Lewis, R. (1996a). A replication study of the structure of the Adolescent Coping Scale: Multiple forms and applications of a self-reported inventory in a counselling and research context. European Journal of Psychological Assessment, 12 (3), 224-235.

Frydenberg, E. \& Lewis, R. (1996b). Social Issues: What concerns young people and how they cope? Peace and Conflict: Journal of Peace Psychology, 2 (3), 271-283.

Frydenberg, E. (1997a). Adolescent coping: Theoretical and research perspectives. Londres: Routledge.

Frydenberg, E. \& Lewis, R. (1997b). Manual: Escalas de afrontamiento para adolescentes (ACS). Madrid: TEA Ediciones.

Frydenberg, E. \& Rowley, G. (1998). Coping with social issues: What australian university students do. Issues in Educational Research, 8, (1), 33-48. 
Frydenberg, E. \& Lewis, R. (1999). Things don't better just because you're older: A case for facilitating reflection. British Journal of Educational Psychology, 69, 81-94.

Hoffman, M.; Levy-Shiff, R. \& Sohlberg, S. (1992). The impact of stress and coping: Developmental changes in the transition to adolescence. Journal of Youth and Adolescence, 21 (4), 451-469.

INEI (1999). Compendio estadístico sociodemográfico 1998/1999. Lima: Editorial Gráfica Monterrico.

Lazarus, R. \& Folkman, S. (1986). Estrés y procesos cognitivos. Barcelona: Martínez Roca.

Muñiz, J. (1996a). Teoría clásica de los tests. Madrid: Pirámide.

Muñiz, J. (1996b). Psicometría. Madrid: Universitas.

Papalia, D. (1990). Psicología del desarrollo. De la infancia a la adolescencia. 3a. edición. México, D.F.: McGraw-Hill.

Sandín, B. (1995). El estrés. En: Belloch, A., Sandín, B. y Ramos, F. (Eds.). Manual de psicopatología. Vol. 2. Madrid: McGraw-Hill Interamericana, 3-52. 


\section{Anexo 1}

\section{Instrucciones}

Los estudiantes suelen tener ciertas preocupaciones o problemas sobre temas diferentes, como la escuela, el trabajo, la familia, los amigos, el mundo en general, etc. En este cuestionario encontrarás una lista de formas diferentes con las que la gente de tu edad suele encarar una gama amplia de problemas o preocupaciones. Deberás indicar, marcando la letra correspondiente, las cosas que tú sueles hacer para enfrentarte a esos problemas o dificultades. En cada afirmación debes marcar con una " $X$ " la letra A, B, C, D o E según creas que es tu manera de reaccionar o de actuar frente a los problemas. No hay respuestas correctas o erróneas. No dediques mucho tiempo a cada frase; simplemente responde lo que crees que se ajusta mejor a tu forma de actuar.

$\begin{array}{ll}\text { Nunca lo hago } & \text { A } \\ \text { Lo hago raras veces } & \text { B } \\ \text { Lo hago algunas veces } & \text { C } \\ \text { Lo hago a menudo } & \text { D } \\ \text { Lo hago con mucha frecuencia E }\end{array}$

Por ejemplo, si algunas veces te enfrentas a tus problemas mediante la acción de "Hablo con otros para saber lo que ellos harían si tuviesen el mismo problema", deberías marcar la C como se indica a continuación:

1. Hablo con otros para saber lo que ellos harían si tuviesen el mismo problema

A

B



D

$E$

1. Hablo con otros para saber lo que ellos harían si tuviesen el mismo problema

2. Me dedico a resolver lo que está provocando el problema

3. Sigo con mis tareas como es debido

4. Me preocupo por mi futuro

5. Me reúno con mis amigos más cercanos

6. Trato de dar una buena impresión en las personas que me importan

7. Espero que me ocurra lo mejor

8. Como no puedo hacer nada para resolver el problema, no hago nada

9. Me pongo a llorar y/o gritar

10. Organizo una acción en relación con mi problema

11. Escribo una carta a una persona que siento que me puede ayudar con mi problema

12. Ignoro el problema

13. Ante los problemas, tiendo a criticarme

14. Guardo mis sentimientos para mí solo(a)

15. Dejo que Dios me ayude con mis problemas

16. Pienso en aquellos que tienen peores problemas, para que los míos no parezcan tan graves

17. Pido consejo a una persona que tenga más conocimiento que yo

\begin{tabular}{|c|c|c|c|}
\hline A & $B$ & C & $D$ \\
\hline$A$ & B & C & $D$ \\
\hline$A$ & B & C & D \\
\hline$A$ & B & C & D \\
\hline$A$ & B & C & $\mathrm{D}$ \\
\hline$A$ & $B$ & C & $D$ \\
\hline$A$ & B & C & $D$ \\
\hline$A$ & B & C & $D$ \\
\hline$A$ & $B$ & C & $D$ \\
\hline$A$ & $B$ & C & $D$ \\
\hline$A$ & B & C & $\mathrm{D}$ \\
\hline$A$ & B & C & $D$ \\
\hline$A$ & B & C & $D$ \\
\hline$A$ & B & C & D \\
\hline$A$ & B & C & $\mathrm{D}$ \\
\hline$A$ & B & C & $D$ \\
\hline A & B & C & D \\
\hline
\end{tabular}


18. Encuentro una forma de relajarme, como oír música, leer un libro, tocar un instrumento musical, ver la televisión, etc.

19. Practico un deporte

20. Hablo con otros para apoyarnos mutuamente

21. Me dedico a resolver el problema utilizando todas mis capacidades

22. Sigo asistiendo a clases

23. Me preocupo por buscar mi felicidad

24. Llamo a un(a) amigo(a) íntimo(a)

25. Me preocupo por mis relaciones con los demás

26. Espero que un milagro resuelva mis problemas

27. Frente a los problemas, simplemente me doy por vencido(a)

28. Intento sentirme mejor bebiendo alcohol, fumando o tomando drogas

29. Organizo un grupo que se ocupe del problema

30. Decido ignorar conscientemente el problema

31. Me doy cuenta que yo mismo(a) me complico la vida frente a los problemas

32. Evito estar con la gente

33. Pido ayuda y consejo para que se resuelvan mis problemas

34. Me fijo en el aspecto positivo de las cosas y trato de pensar en las cosas buenas

35. Busco ayuda o consejo de un profesional para resolver los problemas

36. Salgo y me divierto para olvidar mis dificultades

37. Realizo ejercicios para mantenerme en forma y con buena salud

38. Busco ánimo en otras personas

39. Considero otros puntos de vista y trato de tenerlos en cuenta

40. Trabajo intensamente (Trabajo duro)

41. Me preocupo por lo que está pasando

42. Empiezo, o si ya existe, mejoro la relación con mi enamorado(a)

43. Trato de adaptarme a mis amigos

44. Espero que el problema se resuelva por sí solo

45. Me pongo mal (Me enfermo)

46. Culpo a los demás de mis problemas

47. Me reúno con otras personas para analizar el problema

48. Saco el problema de mi mente

49. Me siento culpable por los problemas que me ocurren

50. Evito que otros se enteren de lo que me preocupa

51 Leo la Biblia o un libro sagrado

52. Trato de tener una visión positiva de la vida

53. Pido ayuda a un profesional

54. Me doy tiempo para hacer las cosas que me gustan

55. Hago ejercicios físicos para distraerme

56. Hablo con otras personas sobre mi problema para que me ayuden a salir de él

57. Pienso en lo que estoy haciendo y por qué lo hago

58. Busco tener éxito en las cosas que estoy haciendo

59. Me preocupo por las cosas que me puedan pasar

60. Trato de hacerme amigo(a) íntimo(a) de un chico o de una chica

61. Trato de mejorar mi relación personal con los demás

62. Sueño despierto que las cosas van a mejorar

A $B \quad$ C $D$ E

A $\quad$ B $\quad$ C $\quad D \quad E$

A $B \quad$ C $\quad D \quad E$

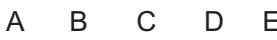

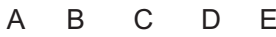

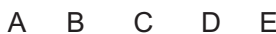

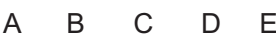

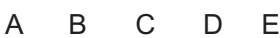

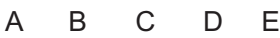

A $B \quad C \quad D \quad E$

A $B \quad C \quad D \quad E$

A $B \quad C \quad D \quad E$

A $B \quad C$ D $E$

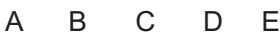

A $B \quad C \quad D \quad E$

A $B \quad C \quad D \quad E$

A B C D E

A $B \quad C \quad D \quad E$

A $B \quad C \quad D \quad E$

A $B \quad C \quad D \quad E$

A $B \quad C \quad D \quad E$

A $B \quad C \quad D \quad E$

A $B$ C C $\quad$ D $\quad E$

A $B \quad C \quad D \quad E$

A $B$ C C $D$ D

A $B \quad C \quad D \quad E$

A $\quad$ B $\quad C \quad$ D $\quad E$

$A \quad B \quad C \quad D \quad E$

A $B \quad C \quad D \quad E$

A $B \quad C \quad D \quad E$

A $B \quad C \quad D \quad E$

A $B \quad C \quad D \quad E$

A $B \quad C \quad D \quad E$

A $B$ C C $\quad D \quad E$

A $B$ B $C$ D

A $B \quad C \quad D \quad E$

A $B \quad C \quad D \quad E$

A $B \quad C \quad D \quad E$

A $B \quad C \quad D \quad E$

A $B \quad C \quad D \quad E$

A $B \quad C \quad D \quad E$

A $B \quad C \quad D \quad E$

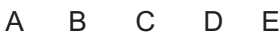

A $B \quad C \quad D \quad E$

A $B \quad C \quad D \quad E$ 
63. Cuando tengo problemas, no sé cómo enfrentarlos

64. Ante los problemas, cambio mis cantidades de lo que como, bebo o duermo

65. Me reúno con las personas que tienen el mismo problema que yo

66. Cuando tengo problemas, me aíslo para poder evitarlos

67. Me considero culpable de los problemas que me afectan

68. Ante los problemas, evito que otros sepan cómo me siento

69. Pido a Dios que cuide de mí

70. Me siento contento(a) de cómo van las cosas

71. Hablo acerca del problema con personas que tengan más experiencia que yo

72. Consigo apoyo de otros, como de mis padres o amigos, para solucionar mis problemas

73. Pienso en distintas formas de enfrentarme al problema

74. Me dedico a mis tareas en vez de salir

75. Me preocupo por el futuro del mundo

76. Procuro pasar más tiempo con la persona con quien me gusta salir

77. Hago lo que quieren mis amigos

78. Me imagino que las cosas van a ir mejor

79. Sufro dolores de cabeza o de estómago

80. Encuentro una forma de aliviar la tensión; por ejemplo, llorar o gritar o beber o tomar drogas
A $\quad$ B $\quad$ C $\quad D \quad E$

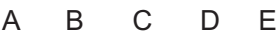

A $B \quad$ C $\quad D \quad E$

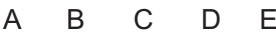

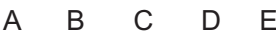

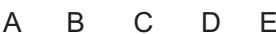

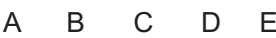

A $\quad B \quad C \quad D \quad E$

A $\quad B \quad C \quad D \quad E$

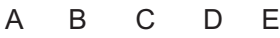

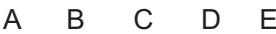

A $B \quad C \quad D \quad E$

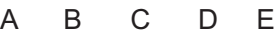

A $B \quad C \quad D \quad E$

A $B \quad C \quad D \quad E$

A $B \quad C \quad D \quad E$

A B C D E

A $B \quad C$ D 


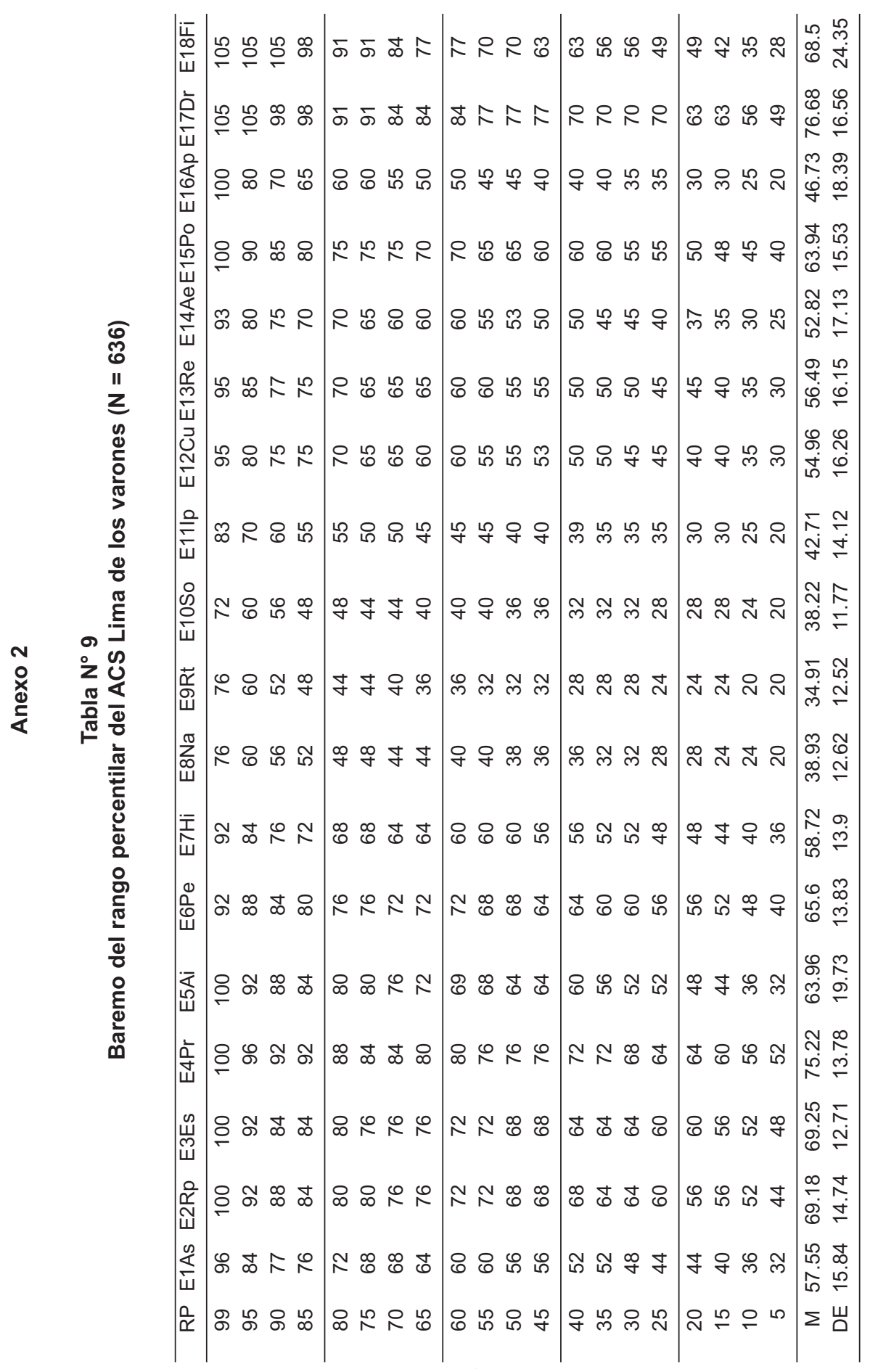




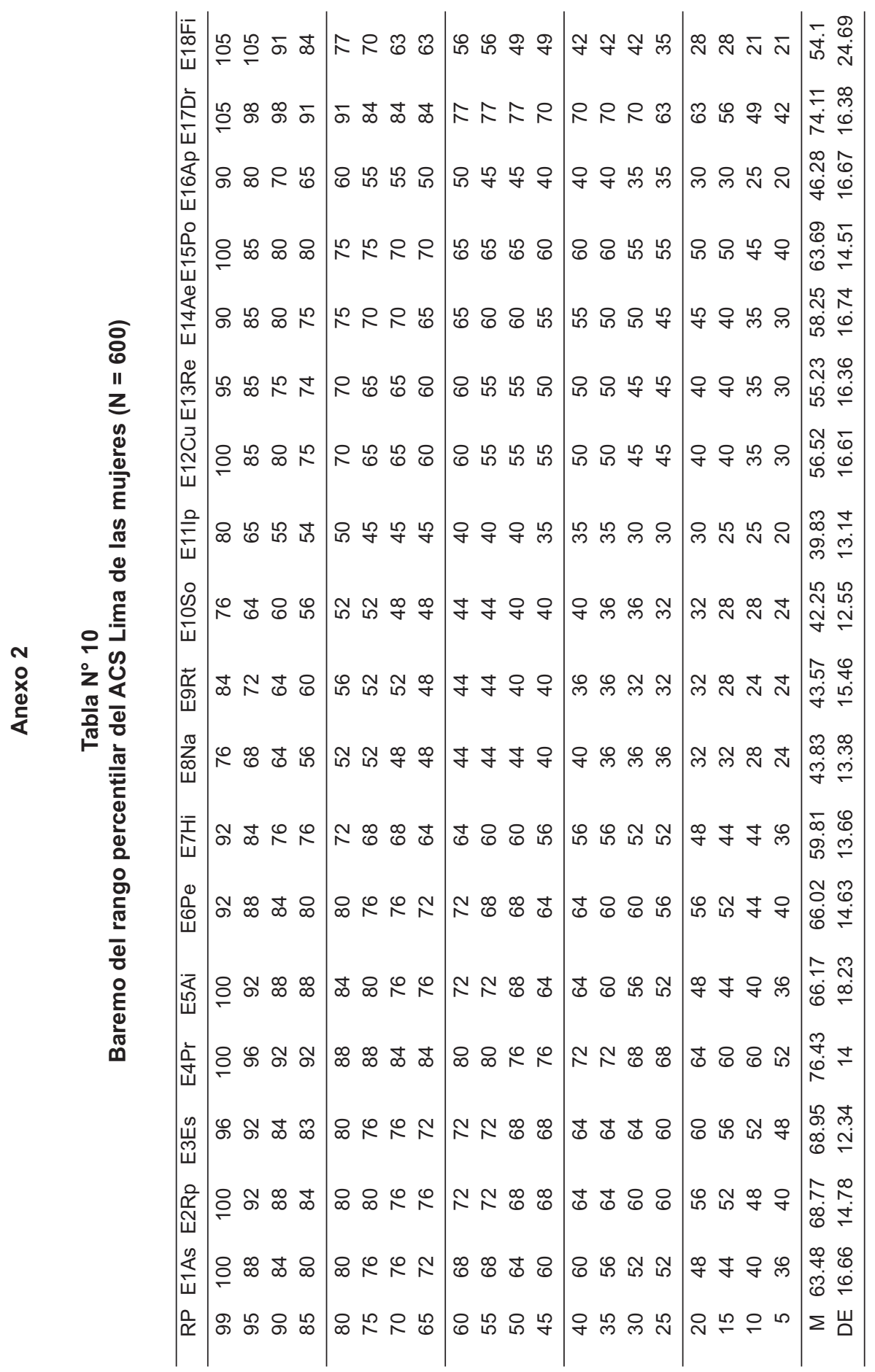

